\title{
Intrinsic properties of a complete sample of HETE-2 gamma-ray bursts
}

\section{A measure of the GRB rate in the Local Universe ${ }^{\star}$}

\author{
A. Pélangeon ${ }^{1}$, J.-L. Atteia ${ }^{1}$, Y. E. Nakagawa ${ }^{2,3}$, K. Hurley ${ }^{5}$, A. Yoshida ${ }^{2,3}$, R. Vanderspek ${ }^{4}$, M. Suzuki ${ }^{14}$, N. Kawai ${ }^{3,6}$, \\ G. Pizzichini ${ }^{7}$, M. Boër ${ }^{8}$, J. Braga ${ }^{9}$, G. Crew $^{4}$, T. Q. Donaghy ${ }^{10}$, J. P. Dezalay ${ }^{11}$, J. Doty ${ }^{4}$, E. E. Fenimore ${ }^{12}$, \\ M. Galassi ${ }^{12}$, C. Graziani ${ }^{10}$, J. G. Jernigan ${ }^{5}$, D. Q. Lamb ${ }^{10}$, A. Levine ${ }^{4}$, J. Manchanda ${ }^{13}$, F. Martel ${ }^{4}$, M. Matsuoka ${ }^{14}$, \\ J.-F. Olive ${ }^{11}$, G. Prigozhin ${ }^{4}$, G. R. Ricker ${ }^{4}$, T. Sakamoto ${ }^{15,20,21}$, Y. Shirasaki ${ }^{16}$, S. Sugita ${ }^{17}$, K. Takagishi ${ }^{18}$, \\ T. Tamagawa ${ }^{3}$, J. Villasenor ${ }^{4}$, S. E. Woosley ${ }^{19}$, and M. Yamauchi ${ }^{18}$
}

(Affiliations can be found after the references)

Received 4 March 2008 / Accepted 19 August 2008

\begin{abstract}
Context. As a result of the numerous missions dedicated to the detection of Gamma-ray bursts (GRBs), the observed properties of these events are now well known. However, studying their parameters in the source frame is not simple since it requires having measurements of both the bursts' parameters and of their distances.

Aims. Taking advantage of the forthcoming Catalog of the High Energy Transient Explorer 2 (HETE-2) mission, the aim of this paper is to evaluate the main properties of HETE-2 GRBs - the peak energy $\left(E_{\text {peak }}\right)$, the duration $\left(T_{90}\right)$ and the isotropic energy $\left(E_{\text {iso }}\right)-$ in their source frames and to derive their unbiased distribution.

Methods. We first construct a complete sample containing all the bursts localized by the Wide-Field X-ray Monitor (WXM) on-board HETE-2, which are selected with a uniform criterion and whose observed parameters can be constrained. We then derive the intrinsic $E_{\text {peak }}, T_{90}$ and $E_{\text {iso }}$ distributions using their redshift when it is available, or their pseudo-redshift otherwise. We finally compute the "volume of detectability" $V_{\max }$ of each GRB, i.e. the volume of the universe in which the burst is bright enough to be part of our sample, and the corresponding number of GRB within their visibility volume $N_{V \max }$, in order to derive a weight for each detected burst accounting both for the detection significance and the star formation history of the universe.

Results. We obtain unbiased distributions of three intrinsic properties of HETE-2 GRBs: $E_{\text {peak }}^{\mathrm{intr}}, T_{90}^{\mathrm{intr}}$ and the isotropic energy of the burst. These distributions clearly show the predominence of X-ray flashes (XRFs) in the global GRB population. We also derive the rate of local GRBs: $R_{0}^{\mathrm{H} 2} \gtrsim$ $11 \mathrm{Gpc}^{-3} \mathrm{yr}^{-1}$, which is intermediate between the local rate obtained by considering only the "high-luminosity" bursts $\left(\sim 1 \mathrm{Gpc}^{-3} \mathrm{yr}^{-1}\right)$ and that obtained by including the "low-luminosity" bursts $\left(\gtrsim 200 \mathrm{Gpc}^{-3} \mathrm{yr}^{-1}\right)$.

Conclusions. This study shows that the XRFs are predominent in the GRB population and are closely linked to the "classical" GRBs. We show that HETE-2 detected no low-luminosity GRB like GRB 980425 or XRF 060218, due to the small size of its detectors, excluding this type of burst from our statistical analysis. The comparison of the GRB rate derived in this study with the known rate of type Ib/c supernovae clearly shows that the progenitors of $\mathrm{SNe} \mathrm{Ib} / \mathrm{c}$ must have some special characteristics in order to produce a gamma-ray burst or an X-ray flash.
\end{abstract}

Key words. gamma rays: bursts - X-rays: bursts

\section{Introduction}

In recent years we have learned that long Gamma-ray bursts $\left(T_{90}>2 \mathrm{~s}\right)$ are associated with the death of massive stars $(M \gtrsim$ 20-30 $M_{\odot}$ ). This origin has been clearly established by the association of a few nearby GRBs with type $\mathrm{Ib} / \mathrm{c}$ supernovae (SNe Ib/c): GRB 980425 detected by Beppo-SAX (Pian et al. 1999) and SN 1998bw (Galama et al. 1998; Kulkarni et al. 1998), GRB 021211 detected by HETE-2 (Crew et al. 2002, 2003) and SN 2002lt (Della Valle et al. 2003), GRB 030329 also detected by HETE-2 (Vanderspek et al. 2003, 2004) and SN 2003dh (e.g. Matheson et al. 2003; Stanek et al. 2003; Hjorth et al. 2003a; Mazzali et al. 2003), GRB 031203 detected by INTEGRAL (Götz et al. 2003) and SN 2003lw

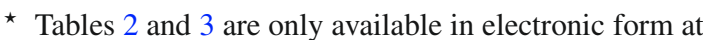
http://www . aanda. org
}

(Malesani et al. 2004), GRB 050525A detected by Swift-BAT (Band et al. 2005) and SN 2005nc (Della Valle et al. 2006a). The association of the nearby GRB 060218 detected by SwiftBAT (Cusumano et al. 2006) with SN 2006aj (Masetti et al. 2006; Modjaz et al. 2006; Pian et al. 2006; Campana et al. 2006; Sollerman et al. 2006; Mirabal et al. 2006b; Cobb et al. 2006) provided a remarkable example of this paradigm. Even though a massive star's explosion can explain the production of both the GRB and the supernova (see for instance the collapsar model proposed by Woosley 1993; see also Woosley \& Bloom 2006; Della Valle 2006a), these two phenomena are profoundly different in nature: the GRB is due to an ultra-relativistic outflow generated by a newborn black hole or magnetar, while the supernova is powered by the radioactive decay of ${ }^{56} \mathrm{Ni}$ within a massive shell of matter ejected at sub-relativistic velocities (e.g. Soderberg et al. 2006c). The question of the link between GRBs 
and $\mathrm{SNe}$ has recently become more complicated by the discovery of two low-redshift, long gamma-ray bursts - GRB 060505 at $z=0.089$ and GRB 060614 at $z=0.125$ - which were not associated with a supernova (Gehrels et al. 2006; Fynbo et al. 2006b; Della Valle et al. 2006b; Gal-Yam et al. 2006). It is thus fair to say that despite very significant advances in our understanding of GRBs, the global picture continues to escape us and key issues remain to be elucidated. Concerning the connection between GRBs and supernovae, the observational clues are based on two facts: the clear association of some GRBs with type Ib/c supernovae, and the evidence, based on statistical studies, that the majority of the 9000 type $\mathrm{Ib} / \mathrm{c}$ supernovae exploding each year in the universe do not produce GRBs. This last point was addressed by Berger et al. (2003), Soderberg et al. (2006a-d, 2004b) and Della Valle (2005, 2006a,c).

While the $\mathrm{SNe} \mathrm{Ib} / \mathrm{c}$ population is rather well sampled in our local environment, the situation is quite different for GRBs, which need a complex series of detections to be fully characterized: detection of the high-energy signal in space, quick transmission to the ground, identification of the afterglow at X-ray, optical or radio wavelengths, and measurement of the redshift. This situation makes our understanding of the GRB population much less secure than for supernovae. Indeed, the observations by Beppo-SAX, HETE-2, and now Swift have demonstrated the diversity of the GRB population, which is composed of classical GRBs, short-duration GRBs, X-ray flashes (XRFs), and lowluminosity GRBs. In this classification, the "classical GRBs" appear to be the "high-luminosity" part (HL-GRBs) of a population which could consist mostly of low-luminosity or subluminous bursts (LL-GRBs), like GRB 980425 (Tinney at al. 1998) and GRB 060218 (Mirabal et al. 2006a), or of X-ray flashes.

In this paper we take advantage of the broad energy coverage of HETE-2 instruments (2-400 keV) to discuss the global properties of the GRB population, and to infer a rate of GRB+XRF events which can be compared with theoretical predictions and with the known rate of type $\mathrm{Ib} / \mathrm{c}$ supernovae. This work is partly based on the forthcoming catalog of HETE-2 GRBs (Vanderspek et al. 2008). For a description of the HETE-2 mission and its instrumentation, see Ricker et al. (2001), Atteia et al. (2003) and Villasenor et al. (2003).

Our work relies on the construction of a complete GRB sample containing all the long-duration bursts localized by the Wide Field X-Ray Monitor (WXM) and having a signal-to-noise ratio $(S N R)$ larger than a given threshold in FREGATE (FREnch GAmma-ray TElescope) or in the WXM. For each burst in this sample, we compute the intrinsic properties at the source $\left(T_{90}\right.$, $E_{\text {peak }}$, and $\left.E_{\text {iso }}\right)$ by correcting the observed properties for the effect of the redshift. When a spectroscopic redshift is not available (62 GRBs out of 82), we use the pseudo-redshift following the method of Pélangeon et al., i.e. the estimate of the redshift which is partly based on both the $E_{\text {peak }}-E_{\text {iso }}$ (e.g. Amati et al. 2002) and $E_{\text {peak }}-L_{\text {iso }}$ (Yonetoku et al. 2004) correlations, and derived from the spectral properties of the prompt emission (for more details see Pélangeon et al. 2006; and Pélangeon \& Atteia 2006). We emphasize that this procedure has little impact on the final results since it introduces an additional uncertainty which is much smaller than the intrinsic dispersion of the parameters under study. In a second step we derive the "visibility distance" for each burst $\left(z_{\max }\right)$, the distance at which the SNR of the burst reaches the threshold of our analysis, and the number of GRB within its visibility volume $\left(N_{V \max }\right)$. We then attribute to each GRB a weight $W=1 / N_{V \max }$, accounting both for the detection significance and the star formation history of the universe. Consequently, this method enables us to renormalize the global distribution, taking into account the true rate of occurrence of each type of GRB. We finally derive the GRB rate detected by HETE-2, and we discuss the relative importance of XRFs and classical GRBs in the overall GRB population.

This paper is organized as follows: Sect. 2 is devoted to the description of the HETE-2 GRB sample. Section 3 references and describes the tools necessary for our study. The three parameters studied here, $E_{\text {peak }}, T_{90}$, and $E_{\text {iso }}$, are discussed in Sects. 4-6 respectively. The remaining three sections are dedicated to the interpretation of the results. In Sect. 7, we check the existence of correlations between the intrinsic parameters. In Sect. 8, we show that our study gives some clues to the nature of $\mathrm{X}$-ray flashes in terms of intrinsic energetics and distance-scales. Finally, in Sect. 9 we discuss the rate of GRBs in the local universe and compare it with previous estimates obtained by other authors. A summary of the main results obtained in this paper is presented in Sect. 10.

\section{Obtaining of a complete sample of HETE-2 bursts}

\subsection{Instrumentation}

Since its launch in October 2000, the HETE-2 satellite (Ricker et al. 2001), dedicated to the detection and observation of Gamma-Ray Bursts, has detected 250 events classified as GRBs (Vanderspek et al. 2008). Thanks to the combination of its 3 instruments, the FREnch GAmma-ray TElescope (FREGATE, Atteia et al. 2003), the Wide-field X-ray Camera (WXM, Shirasaki et al. 2003) and the Soft X-ray Camera (SXC, Villasenor et al. 2003), HETE-2 has a broad energy range covering the hard $X$-rays and $\gamma$-rays $(2-400 \mathrm{keV})$, allowing the detection of both classical and soft GRBs (XRFs). Moreover, HETE-2 was designed to provide GRB positions to the community through the GRB Coordinates Network (GCN) (Barthelmy et al. 2000) with an accuracy of one to several arcminutes, within seconds of the trigger. Consequently, multi-wavelength ground follow-up was done for most HETE-2 GRBs, leading to the determination of 25 spectroscopic redshifts.

\subsection{Burst selection}

It is of prime importance for our study to construct a GRB sample for which the detection criteria are fully understood and can be reproduced. This is essential for the determination of the visibility volume of each GRB in the sample. In addition it is necessary to be able to compute for each burst the three parameters of our study: $E_{\text {peak }}, T_{90}$, and the fluence. This section explains the construction of the sample ${ }^{1}$.

\subsubsection{Availability of the spectral data}

We considered all the 250 events that constitute the total sample detected by HETE-2, i.e. both triggered and untriggered bursts, thus reducing the bias due to trigger algorithms. Then, we selected the bursts for which the angle of incidence was measured by any experiment. Its knowledge is necessary to perform the spectral analysis of the bursts and to derive their $E_{\text {peak }}$ and $E_{\text {iso. }}$. Of the 250 GRBs, 132 have no accurate localization and were rejected, leaving 118 GRBs.

Among these bursts, we have also rejected GRB 040810 because this long and intense burst occurred as the instruments

\footnotetext{
1 The order of the steps described in this section is not important; we could have performed them in any order and obtained the same sample.
} 
were being shut down. Consequently only the precursor was detected by HETE-2.

\subsubsection{Technical problems}

Of the remaining 117 GRBs, 2 bursts were rejected due to technical problems: GRB 010110 was detected and localized by the WXM when FREGATE was not working. It is thus impossible to constrain the spectral parameters with only the WXM data. The second burst rejected is GRB 021113. At the moment of its detection there were onboard software problems that did not permit the trigger to reach the ground. The spectral data are thus not available.

\subsubsection{WXM localization}

Considering the 115 remaining GRBs with a known angle, we removed 24 GRBs for which the angle was obtained through the localization by the Inter Planetary Network (IPN) or the Swift satellite (Gehrels et al. 2004). Indeed, as the IPN and Swift have different sensitivities, we would have added a bias to our sample by considering them.

Moreover, in order to have bursts well within the field of view of the WXM, we have considered an "incident angle limit" of $45^{\circ}$. This angle corresponds to a limit of detection for the WXM (Shirasaki et al. 2003), and to a decrease of the effective area for FREGATE (Atteia et al. 2003). With this cut, only one burst is rejected: GRB 020201, whose angle is $55^{\circ}$. This step leaves 90 GRBs in the sample (see Fig. 1).

\subsubsection{Short-duration bursts}

We also rejected the two bursts classified without ambiguity as short-duration bursts: GRB 020531 (Lamb et al. 2003) and GRB 050709 (Villasenor et al. 2005). This is based on the fact that this class of burst is probably not associated with the same progenitors as the long GRBs. As one goal of this paper is to discuss the rate of long GRBs in the local universe and to compare it with the rate of $\mathrm{SNe}$, we have excluded the short-duration bursts, leaving 88 bursts in the sample.

\subsubsection{Threshold cut}

Finally, in order to have homogeneous detection criteria for all the events of the sample, we removed the GRBs with a signalto-noise ratio $(S N R)$ lower than a given threshold in FREGATE and in the WXM. These SNR were computed as follows:

- we measured the peak count rate of each burst. For FREGATE we used two different time resolutions (1.3 and $5.2 \mathrm{~s}$ ) and two different energy ranges (6-80 and $30-400 \mathrm{keV}$ ) corresponding to the bands used by the instrument to trigger. For the WXM we used three different time resolutions $(1.2,4.9$ and $9.8 \mathrm{~s})$ and the total energy range of the WXM $(2-25 \mathrm{keV})$;

- we computed a $S N R$ for each burst in all the combinations of time resolution and energy range. We kept only the GRBs for which the SNR exceeded 7.9 in FREGATE or 5.8 in the WXM (in at least one combination of the time and energy ranges, see Fig. 2).

This last criterion removed 6 bursts with a low $S N R$ : GRB 011103, XRF 020903, GRB 030323, GRB 030706, GRB 040131 and GRB 040228.

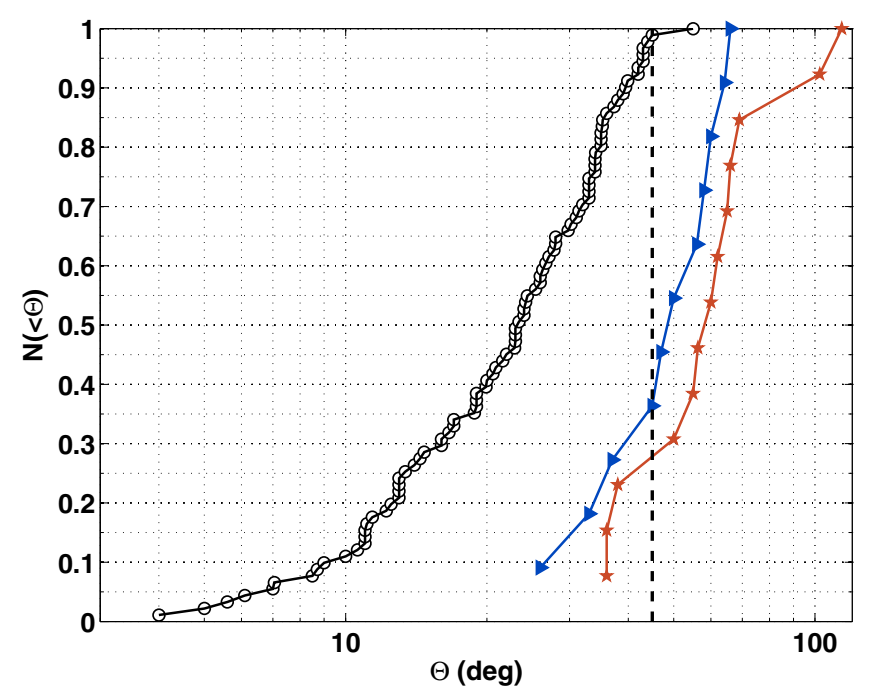

Fig. 1. Boresight angle cumulative distribution of the 115 GRBs localized by HETE-2/WXM, the Interplanetary Network or Swift. The dark dashed vertical line represents the "angle limit" of $45^{\circ}$, corresponding to the limit of detection by the WXM. The GRBs localized by the WXM are represented with black open circles, the ones localized by the IPN are in blue filled triangles, and the ones localized by Swift are in darkred filled stars.

Our final sample contains 82 GRBs whose properties are given in Tables 2 and 3.

\subsection{The cases of XRF 020903 and GRB 030323}

These two bursts have a spectroscopic redshift but they are rejected from our sample due to their SNR just below the thresholds of FREGATE and the WXM (Fig. 2). As they have spectroscopic redshifts $-z=0.25$ for XRF 020903 (Soderberg et al. 2004a) and $z=3.372$ for GRB 030323 (Vreeswijk et al. 2003) - we can argue that even if they had been present in our sample, the results presented in this paper would actually have been reinforced. Indeed, situated at a moderately high redshift, GRB 030323 has a large visibility volume and thus a low weight. On the other hand, the nearby XRF 020903 has a small visibility volume and hence a large weight. Since this is an XRF with a low $E_{\text {peak }}$, adding it to our GRB sample would have increased the predominance of X-ray flashes which is shown in Sect. 4, and strengthened the conclusions of this paper.

\section{Deriving the intrinsic properties of HETE-2 GRBs}

\subsection{Burst parameters}

We have used the spectral parameters and durations available in the HETE-2 Catalog (Vanderspek et al. 2008) which summarizes the information on GRBs detected by HETE-2 during the entire mission and thus complements the previous spectral analyses based on smaller samples of HETE-2 GRBs performed by Barraud et al. (2003, 2004) and Sakamoto et al. (2005).

\subsection{Burst distance}

At the end of the 90s, the growing sample of GRBs with measured redshift allowed new types of studies: searches for correlations between physical quantities - characterizing the gamma-ray bursts' light-curves and/or spectra - in their rest frame. Following the discovery of correlations between $E_{\text {iso }}$, 


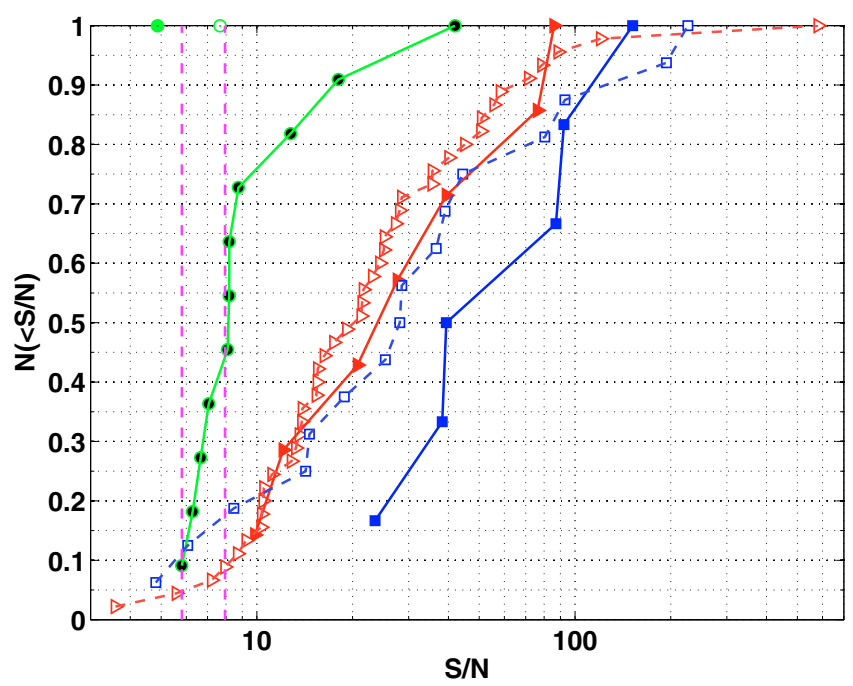

Fig. 2. Signal-to-noise ratio $(S N R)$ cumulative distribution of the 88 GRBs localized by the WXM and within $45^{\circ}$ of the boresight. The threshold of FREGATE $(S N R=7.9)$, is represented with the rightmost vertical magenta dashed line, whereas the threshold of the WXM $(S N R=5.8)$ is represented with the left-most vertical magenta dashed line. The different symbols show the configuration of time resolution and energy range leading to the highest $S N R$ for each burst: a given shape is associated with a given energy range and a given color corresponds to a given time resolution: [WXM/2-25 keV/1.2 s] = lightgreen filled circles; [WXM/2-25 keV/4.9 s] = light-green open circles; [WXM/2-25 keV/9.8 s] = dark filled circles; [FREG $/ 6-80 \mathrm{keV} / 1.3 \mathrm{~s}$ ] = red filled triangles; [FREG/6-80 keV/5.2 s] = red open triangles; [FREG/30-400 keV/1.3 s] = blue filled squares; [FREG/ $30-400 \mathrm{keV} / 5.2 \mathrm{~s}]=$ blue open squares. Note that there is only one light-green filled circle and one light-green open circle (left part of the figure) due to the fact that only one burst has its highest $S N R$ in the configuration $[\mathrm{WXM} / 2-25 \mathrm{keV} / 1.2 \mathrm{~s}$ ] and one burst in the configuration [WXM/2-25 keV/4.9 s].

the isotropic equivalent energy emitted by the burst, and various intrinsic GRB properties, it has been suggested that some GRB observables could be used as luminosity indicators, and hence as redshift indicators. The most used are the lag-luminosity $\left(\tau_{\mathrm{lag}}-L_{\mathrm{p}, \text { iso }}\right)$ correlation (Norris et al. 2000), the variability-luminosity $\left(V-L_{\mathrm{p}, \text { iso }}\right)$ correlation (e.g. Fenimore \& Ramirez-Ruiz 2000; Reichart et al. 2001; Lloyd-Ronning et al. $2002 \mathrm{a})$ and the $E_{\text {peak }}-E_{\text {iso }}$ correlation - suggested by several authors (see e.g. Lloyd et al. 2000; Atteia 2000; Lloyd-Ronning \& Ramirez-Ruiz 2002b) - before Amati et al. (2002) firmly established it with a study of 12 GRBs with known redshift. Other correlations were found later, such as the $E_{\text {peak }}-L_{\mathrm{p} \text {,iso }}$ correlation (Yonetoku et al. 2004), the $E_{\text {peak }}-E_{\gamma}$ correlation (Ghirlanda et al. 2004) and the $E_{\text {peak }}-L_{\mathrm{p}, \text { iso }}-T_{0.45}$ correlation (Firmani et al. 2006). Recently, in his construction of the Hubble Diagram from a sample of 69 GRBs with redshifts, Schaefer (2007) showed that the use of five of these correlations simultaneously - lag-luminosity ( $\tau_{\text {lag }}-L_{\mathrm{p} \text {,iso }}$, Norris et al. 2000); $V-L_{\mathrm{p} \text {,iso }}$ (Fenimore \& Ramirez-Ruiz 2000); $E_{\text {peak }}-L_{\text {p,iso }}$ (Schaefer 2003); $E_{\text {peak }}-E_{\gamma}$ (Ghirlanda et al. 2004) and the minimum light-curves rise time versus luminosity $\left(\tau_{\mathrm{RT}}-L_{\mathrm{p} \text {,iso }} \text {, Schaefer } 2002\right)^{2}-$ led to more reliable luminosity distances than the use of only one distance indicator.

The design of HETE-2 permitted the study of the energetics at work in GRBs, both in the observer and in the source

\footnotetext{
2 See e.g. Ghirlanda et al. (2006) or Schaefer (2007) for a complete summary of these correlations.
}

frames. For example, the $E_{\text {peak }}-E_{\text {iso }}$ correlation (Amati et al. 2002) was confirmed and extended at lower energy, thanks to the sample of HETE-2 X-ray rich GRBs (XRRs) and X-ray flashes (Amati 2003; Lamb et al. 2004a,b; Amati 2006), particularly XRF 020903 (Sakamoto et al. 2004). The HETE-2 sample is thus appropriate for the determination of pseudo-redshifts based on the $E_{\text {peak }}-E_{\text {iso }}$ correlation. A first attempt in this direction was proposed by Atteia (2003), which was later revised by Pélangeon et al. (2006) to take into account the complex lightcurves of some long GRBs ${ }^{3}$.

The accuracy of this redshift indicator and its use in this study is discussed in the following paragraph.

\subsection{Justification of the pseudo-redshifts used in this study}

As shown in Table 3, 18 GRBs contained in our sample have a spectroscopic redshift and 2 have a photometric redshift (GRB 020127 and GRB 030115). We computed a pseudoredshift for the remaining 62 (among the 20 bursts having a known redshift, 14 were used for calibrating the pseudo- $z$ ).

In order to test the impact of the use of pseudo-redshifts in this work:

- we computed the pseudo-redshifts of the 20 GRBs having a spectroscopic or photometric redshift;

- for these same 20 GRBs we computed the ratio between the pseudo-redshift and the redshift, and the ratio of the pseudointrinsic properties derived with pseudo-redshifts to the intrinsic properties derived using the redshift (Fig. 3).

We note that the dispersion of the ratio between the pseudoredshifts and the redshifts is smaller than a factor of 2, except for two outliers: GRB 020819 and GRB 051022 for which the spectroscopic redshift differs from the pseudo-redshift by factors of 2.95 and 2.15 respectively. For these $20 \mathrm{GRBs}$, the dispersion between the luminosity distance estimated with the pseudo-redshifts $\left(D_{\mathrm{L}, \text { estim }}\right)$ and the luminosity distance measured with the redshifts $\left(D_{\mathrm{L} \text {,meas }}\right)$ is: $\sigma_{\mathrm{D}_{\mathrm{L}}}=\log \left(D_{\mathrm{L}, \text { estim }} / D_{\mathrm{L} \text {,meas }}\right)=$ 0.125 dex, i.e. a factor of $1.34^{4}$.

The dispersion is also smaller than a factor of 2 for the $E_{\text {peak }}$ (Fig. 3, top-right panel) and the $T_{90}$ parameters (Fig. 3, bottom-left panel). This is not surprising since these two quantities vary as $(1+z)$, reducing the impact of the redshift errors. $E_{\text {iso }}$, which is more redshift-dependent than $E_{\text {peak }}$ and the duration, is more scattered and its dispersion is larger than a factor of 2. This corresponds to the bursts having the highest difference between their spectroscopic redshift and their pseudoredshift: GRB 020819, GRB 040912B and GRB 051022 have a pseudo- $z$ estimate two to three times higher than their redshift; and GRB 020124, GRB 030115 and GRB 050408 for which the pseudo- $z$ is lower than the spectroscopic redshift by a factor of 2 (Fig. 3, bottom-right panel).

We consider that the good agreement of pseudo- $z$ with spectroscopic redshifts for the 20 GRBs having a measured $z$ is encouraging. One may question however if the pseudo- $z$ values computed without knowing the spectroscopic redshift are as accurate as those computed when the redshift is known - i.e. do pseudo- $z$ have predictive power? - Unfortunately, most of the bursts for which we have computed a pseudo- $z$ do not have redshift measurements, so comparison with the redshift-estimates cannot be done. However, in some cases the pseudo- $z$ values

\footnotetext{
3 See also http: //www . ast. obs-mip. fr/grb/pz

4 If we do not consider the two outliers GRB 020819 and GRB 051022 the dispersion is $\sigma_{D_{\mathrm{L}}}=0.089$ dex, i.e. a factor of 1.23 .
} 


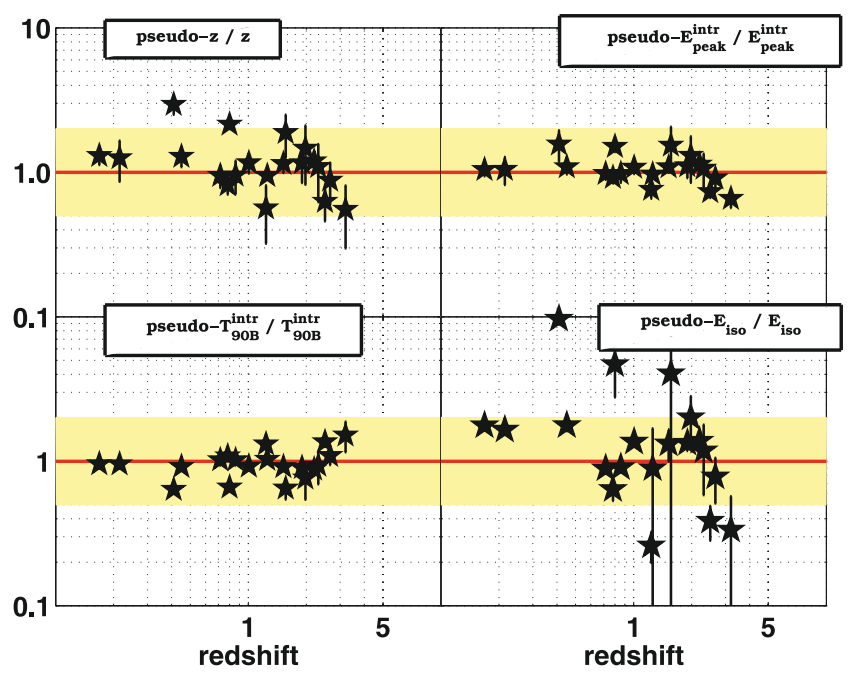

Fig. 3. Ratio between the pseudo-redshift and the redshift for the 20 GRBs contained in our sample for which both quantities are available (upper-left panel). The ratio of the 3 pseudo-intrinsic and intrinsic parameters studied are also shown, respectively $E_{\text {peak }}$ (upper-right panel), $T_{90}$ (lower-left panel) and $E_{\text {iso }}$ (lower-right panel). In all plots, the filled region corresponds to a deviation from the equality between the two parameters by a factor of 2 , and the error bars are at the $1 \sigma$ level (stat.+syst.)

we issued in GCN Circulars were followed by a spectroscopic measurement. For instance, we determined the pseudo-redshifts of GRB $050525^{5}$ and GRB $070125^{6}$ before their redshifts were known. The spectroscopic measurements have confirmed our estimates. Moreover, two other GRBs detected in 2003 and 2004 by HETE-2 had a pseudo-redshift (Pélangeon et al. 2006) but no redshift. Some later observations of the host galaxies resulted in the determination of their spectroscopic redshifts, in agreement with their pseudo-redshifts: Rau et al. (2005) measured the redshift of the host galaxy of GRB 030531 to be $z=0.782 \pm$ $0.001(\hat{z}=0.64 \pm 0.15$, Pélangeon et al. 2006), and Stratta et al. (2007) obtained the redshift of the X-ray flash 040912: $z=1.563 \pm 0.001$ from the [OII] line of the host-galaxy spectrum, also consistent with its previously determined pseudoredshift $(\hat{z}=2.90 \pm 1.60$, Pélangeon et al. 2006).

In order to quantify the effect of the uncertainty from the pseudo-redshifts on the intrinsic distributions, we performed the following test: we artificially increased the errors on the pseudo$z$ values which are taken into account in the construction of the intrinsic parameters distributions (see Sect. 4.3). This extra dispersion takes on three values corresponding to factors of 1.4, 2.0

5 The first GCN Circular containing the computation of a pseudoredshift in nearly real time was done for this burst, using the spectral parameters obtained by Konus-WIND (Golenetskii et al. 2005a). We found $\hat{z}=0.36 \pm 0.10$ (Atteia \& Pélangeon 2005a) whereas the spectroscopic redshift obtained by spectroscopy of the host galaxy and published the day after was $z=0.606$ (Foley et al. 2005). The difference between the pseudo-redshift and the redshift was due to an incorrect published fluence (Golenetskii et al. 2005b). As soon as the correct value was available, we recomputed the pseudo-redshift of this burst and found $\hat{z}=0.64 \pm 0.10$ (Atteia \& Pélangeon 2005b).

${ }^{6}$ We have computed a redshift-estimate of this bright burst using the spectral parameters derived from two different instruments, Rhessi $(\hat{z}=$ $1.63 \pm 0.80$, Pélangeon \& Atteia 2007a) and Konus-WIND $(\hat{z}=1.34 \pm$ 0.30 , Pélangeon \& Atteia 2007b). Both values were consistent with a redshift of $\sim 1.5$. The spectroscopic redshift $z \sim 1.54$ was measured several days after our estimate (Fox et al. 2007; see also Cenko et al. 2008). and 3.0. We tested the impact of this extra dispersion on the intrinsic $E_{\text {peak }}$ distributions ("simple" and "unbiased"), and thus we show that even if the pseudo- $z$ were only accurate to a factor of 2 or 3 , this would not significantly change the intrinsic parameter distributions and all the subsequent results obtained in this paper (see Sect. 4).

It was recently proposed by Butler et al. (2007) that the correlations between the intrinsic GRB parameters found in the preSwift era were due to instrumental biases (e.g. detector thresholds effects) rather than to real physical properties of the sources. This is the result of a study they performed on a sample of 77 Swift GRBs with measured redshifts, where they found that those GRBs are on average harder than the pre-Swift measurements, i.e. they have higher $E_{\text {peak }}$ and lower $E_{\text {iso }}$. As a consequence, they conclude that these correlations cannot be used to estimate the GRB redshifts.

Since no clear conclusion can be drawn from this debate, we have also considered this possibility and we calculated all the distributions twice: one case where we attribute a pseudoredshift to the 62 bursts which have no redshift, and the other case where we randomly attribute one of the 24 secure redshifts measured for the HETE-2 long GRBs. Therefore, for all the distributions we obtain we will comment on how they change between the two cases.

Throughout the paper we use the following notations:

- the spectroscopic or photometric redshifts are denoted by $z$;

- the pseudo-redshifts are denoted by $\hat{z}$;

- $z_{\text {sample }}$ refers to the value of the redshift used in our study: this is the spectroscopic/photometric redshift $(z)$ when it is available and the pseudo-redshift ( $\hat{z}$ ) otherwise (see Table 2 ).

\section{Parameter 1: $E_{\text {peak }}$}

\subsection{Measuring the $E_{\text {peak }}$ of $X$-ray flashes}

The peak energy of a burst is generally measured using the XSPEC software (Arnaud \& Dorman 2003) and fitting a phenomenological model - GRB (Band) Model (Band et al. 1993) or Cutoff Power Law Model - on the spectral data. However, for some X-ray flashes, the peak energy is lower than $10 \mathrm{keV}$, so the data do not constrain the four parameters of the Band function. For those bursts, the value obtained is too close to the lower boundary of FREGATE $(6 \mathrm{keV})$ and of the WXM $(2 \mathrm{keV})$. In this case, we observe a power-law spectrum instead of a Band function. To solve this problem, Sakamoto et al. (2004) showed that a modified function, called the Constrained Band Model $(C B M)$, based on a three-dimensional subspace of the full fourdimensional Band function parameter space, could be used. We adopted this model to fit the data when it was necessary (see Table 2).

However, in order to check whether such a model could add a bias to the estimate of $E_{\text {peak }}$, we used XSPEC to simulate an $\mathrm{XRF}$ spectrum as follows:

- we use the data of a real XRF, in order to have real background statistics and instrumental response matrices;

- we construct a fake burst that follows a Band model with typical values of -1.0 and -2.3 for the power-law indices at low and high energy respectively. These parameters correspond to the mean values for GRB spectra according to BATSE results (see e.g. Preece et al. 2000; Kaneko et al. 2006). The two other parameters, $E_{\text {peak }}$ and the normalization, are the variable parameters that let us produce fake XRFs of greater or lesser intensity; 
- we generate fake data files for XRFs of various brightnesses and various $E_{\text {peak }}$;

- we then perform a spectral analysis using a Band model and a Constrained Band model, and compare the parameters obtained.

We find that the Band model is valid for $E_{\text {peak }}$ down to $8 \mathrm{keV}$. For this limiting value, the errors on the spectral parameters are slightly larger than those obtained using the Constrained Band function, but they are still valid. For $E_{\text {peak }}$ below $8 \mathrm{keV}$, the Constrained Band model is preferable: it gives correct $E_{\text {peak }}$ values or provides at least reliable upper limits.

In all cases we obtain consistent values between the CBM and the Band function when both functions are applicable, and these values are consistent with the ones we introduced to produce the fake XRFs. We thus conclude that the values determined with the Constrained Band model are not biased.

\subsection{The observed $E_{\text {peak }}$ distribution}

Several points can be noticed in Fig. 4: first, the $E_{\text {peak }}^{\text {obs }}$ distribution peaks at about $100 \mathrm{keV}$ and extends over the entire energy range of HETE-2 (2-400 keV). Second, the two classes of bursts (classical GRBs and X-ray flashes) are clearly distinguished. As a comparison, the study of Kaneko et al. (2006) on the complete spectral catalog of bright BATSE Gamma-Ray Bursts, leads to an $E_{\text {peak }}^{\text {obs }}$ distribution peaking at about $300 \mathrm{keV}$, extended at high energies up to several $\mathrm{MeV}$ and without significant events at very low energies. The paucity of soft $(\sim \mathrm{a}$ few $\mathrm{keV})$ and hard $(\sim \mathrm{MeV})$ bursts in our sample is probably not real. For the soft bursts, this is clearly visible by a simple comparison of the BATSE and HETE-2 $E_{\text {peak }}$ distributions. As BATSE triggers were generally done on the count rate between 50 and $300 \mathrm{keV}$, the ratio between the XRFs and the GRBs is low. For HETE-2, the energy range extends down to $2 \mathrm{keV}$ and enables the detection of more XRFs but still prevents the detection of XRFs with $E_{\text {peak }}$ below a few $\mathrm{keV}$. As far as the hard tail of the $E_{\text {peak }}$ distribution is concerned, the low sensitivity of the detectors at high energy is also one of the causes of the inefficient detection of hard bursts. Moreover, two bursts emitting the same energy with different hardness do not have the same number of photons: the softer GRB has more photons than the harder one. Consequently, BATSE-like or HETE2-like missions are probably not able to derive a true observed $E_{\text {peak }}$ distribution. The distributions obtained clearly show that the $E_{\text {peak }}^{\text {obs }}$ distribution depends strongly on the energy range of the instruments involved.

\subsection{The intrinsic $E_{\text {peak }}$ distribution}

So far, the intrinsic $E_{\text {peak }}$ distribution has been very little discussed, mainly due to the difficulty of measuring both the $E_{\text {peak }}$ and the redshift of the bursts. On the one hand, BATSE, BeppoSAX and HETE-2 did constrain the $E_{\text {peak }}^{\text {obs }}$ thanks to their wide energy ranges, but the bursts they detected suffered from a lack of redshift determination. On the other hand, thanks to its performance and fast localization capability, Swift currently enables more redshift measurements, but the narrow energy range of the BAT instrument (15-150 keV) does not allow a good constraint of the $E_{\text {peak }}^{\text {obs }}$.

As mentioned by Amati (2006), about 70 intrinsic $E_{\text {peak }}$ are available, but this sample contains bursts detected by different satellites. Our work is based on a sample containing all the bursts

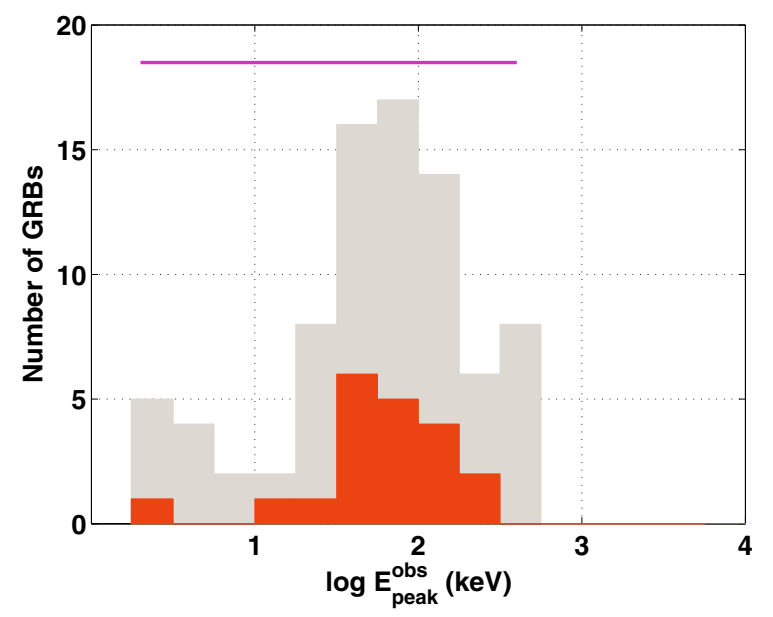

Fig. 4. Distribution of the $E_{\text {peak }}^{\text {obs }}$ measured for the complete sample of 82 GRBs detected and localized by HETE- 2 . The sub-sample's histogram of bursts with secure redshifts $(z)$ is shown in red, and the total sample's histogram is shown in grey. The bursts on the left part of the histogram correspond to the events that have a measured higher limit in $E_{\text {peak }}^{\text {obs }}$ of about $4 \mathrm{keV}$ (see Table 2). The energy range of HETE-2 $(2-400 \mathrm{keV})$ is symbolized by the magenta horizontal solid line.

localized with a single satellite, hence avoiding selection effects inherent to the use of data from different instruments.

We see in Fig. 5 that the $E_{\text {peak }}^{\text {intr }}$ distribution is broader than the $E_{\text {peak }}^{\text {obs }}$ distribution. At high energies, some bursts have an $E_{\text {peak }}^{\text {intr }}$ reaching a few $\mathrm{MeV}$. Nevertheless, as we previously mentioned, the energy range of HETE-2 (2-400 keV) prevents us from drawing any conclusion about the high energy part of the $E_{\text {peak }}$ distribution.

At low energies, bursts having an $E_{\text {peak }}^{\text {obs }}$ lower than $20 \mathrm{keV}$, and defined as X-ray flashes in the observer frame, still have a low $E_{\text {peak }}$ in their source frame. For most of those GRBs, the low intrinsic peak energy is due to their intrinsic faintness as they are at low or intermediate redshift. (This point is discussed in more detail in Sect. 8)

Joining these two extreme energy ranges, the intrinsic $E_{\text {peak }}$ distribution of HETE-2 GRBs extends over 3 decades in energy. The true width of the distribution is probably even larger because bursts with $E_{\text {peak }}$ lower than $1 \mathrm{keV}$ are not detected, and bursts with $E_{\text {peak }}$ greater than $1 \mathrm{MeV}$ cannot be constrained by an HETE2-like mission.

\subsection{The unbiased $E_{\text {peak }}$ distribution}

\subsubsection{Construction}

In order to derive the true $E_{\text {peak }}$ distribution, the distance-scale is not the only correction that has to be applied to the observed $E_{\text {peak }}$ distribution. All the bursts do not have the same brightness and are not detected at the same significance in the observer frame. We took this into account to correct the intrinsic $E_{\text {peak }}$ distribution obtained in the previous section.

To do this we used the following method:

(1) in order to take into account the errors on all the parameters used (spectral properties and redshift) we have computed "smoothed distributions" by:

- producing for each burst a set of $100 E_{\text {peak,sim }}^{\text {obs }}$ randomly selected within the $90 \%$ confidence level (c.1.) 


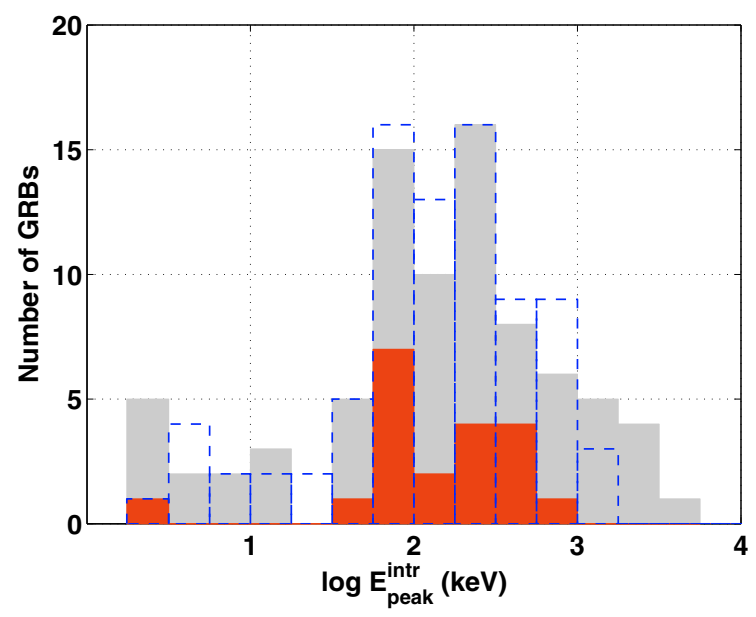

Fig. 5. Distribution of $E_{\text {peak }}^{\text {intr }}$ for the complete sample of 82 GRBs detected and localized by HETE-2. The histogram related to the subsample of bursts with secure redshifts $(z)$ is shown in red, and the total sample's histogram is shown in grey. The blue dashed histogram corresponds to the studied case 2 (see Part 3.3), i.e. the sample of 62 GRBs without secure redshift that are randomly given a redshift from the HETE-2 redshift distribution.

error range of the measured $E_{\text {peak }}^{\text {obs }}$ given in Table $2^{7}$ and 100 redshifts randomly selected within the $90 \%$ c.l. error range of their corresponding spectroscopic/photometric redshift or pseudo-redshift $z_{\text {sim. }}$. Combined with the 100 simulated $E_{\text {peak,sim, }}^{\text {obs }}$, we thus obtained a sample of 100 simulated $E_{\text {peak,sim; }}^{\text {intr }}$;

- representing each of the 8200 simulated bursts (b) with a normal distribution of the logarithmic $E_{\text {peak }}^{\text {intr }}$ values $\left(L E_{\mathrm{p}, b}^{\mathrm{intr}}\right)$ :

$f_{b}\left(l e_{\mathrm{p}, i}\right)=\frac{1}{\sigma_{L E_{\mathrm{p}, b}^{\text {int }}}} \times \exp \left[-\frac{1}{2}\left(\frac{l e_{\mathrm{p}, i}-\mu_{L E_{\mathrm{p}, b}^{\mathrm{int}}}}{\sigma_{L E_{\mathrm{p}, b}^{\text {intr }}}}\right)^{2}\right]$

where $l e_{\mathrm{p}, i}$ is the variable of the functions $f_{b}, \mu_{L E_{\mathrm{p}, b}^{\mathrm{intr}}}$ is the base $10 \operatorname{logarithm}$ of $E_{\text {peak,sim }}^{\text {intr }}$ related to the burst $b$ and $\sigma_{L E_{\mathrm{p}, b}^{\mathrm{int}}}$ is set to 0.05 ;

(2) then, we computed for the 8200 simulated GRBs a weight related to their detection significance. For that, we:

- determine the maximum redshift $z_{\max }$ at which the source could have been detected by the instruments, by first comparing the $S N R$ computed in Sect. 2 (see Table 2) to the SNR threshold of the HETE-2 instruments, and then by redshifting the sources until their peak photon flux reaches the SNR threshold of the trigger instrument, giving us both the visibility distance between the source and the satellite and the maximum redshift $z_{\max }$;

- assume that the GRB rate follows the star formation rate. For this we have adopted the model $\mathrm{SFR}_{2}$ of Porciani \& Madau (2001) that reproduces a fast evolution between $z=0$ and $z=1$ and remains constant beyond $z \geq 2$

$R_{\mathrm{SFR}_{2}}(z) \propto 0.15 h_{65} \frac{\exp (3.4 z)}{\exp (3.4 z)+22}$

\footnotetext{
${ }^{7}$ For some XRFs, the lower error limit could not be constrained. In this case the value was set to $E_{\text {peak,min }}^{\text {obs }}=1 \mathrm{keV}$. For some other GRBs, it was the upper error limit which was not constrained. In this case we set the value to $E_{\text {peak,max }}^{\text {obs }}=1 \mathrm{MeV}$.
}

- derive for each burst the number of GRB per year within its visibility volume

$$
N_{V \max } \propto \int_{0}^{z_{\max }} \mathrm{d} z \frac{\mathrm{d} V(z)}{\mathrm{d} z} \frac{R_{S F R_{2}}(z)}{1+z} .
$$

In this equation $\mathrm{d} V(z) / \mathrm{d} z$ is the comoving element volume, described by

$\frac{\mathrm{d} V(z)}{\mathrm{d} z}=\frac{c}{H_{0}} \frac{4 \pi \mathrm{d} l^{2}(z)}{(1+z)^{2}\left[\Omega_{\mathrm{M}}(1+z)^{3}+\Omega_{K}(1+z)^{2}+\Omega_{\Lambda}\right]^{1 / 2}}$

where $H_{0}$ is the Hubble constant, $\Omega_{K}$ is the curvature contribution to the present density parameter $\left(\Omega_{K}=\right.$ $\left.1-\Omega_{\mathrm{M}}-\Omega_{\Lambda}\right), \Omega_{\mathrm{M}}$ is the matter density and $\Omega_{\Lambda}$ is the vacuum density. Throughout this paper we have assumed a flat $\Lambda \mathrm{CDM}$ universe where $\left(H_{0}, \Omega_{\mathrm{M}}, \Omega_{\Lambda}\right)=$ (65 $\left.h_{65} \mathrm{~km} \mathrm{~s}^{-1} \mathrm{Mpc}^{-1}, 0.3,0.7\right)$.

This procedure allows us to give each burst a weight $\left(W_{b}\right)$ inversely proportional to $N_{V \max }$. The rationale of weighting each burst by $1 / N_{V \max }$ is the following: the visibility volume is different for each GRB of our sample. Moreover, each burst observed is randomly taken from all the bursters present in its visibility volume. In this way, rare bright bursts, having a large visibility volume, will have low weights, while faint local GRBs will have heigher weights. This procedure also takes into account the fact that the GRB rate evolves with redshift, leading to the fact that GRBs are about ten times more frequent at $z \sim 1$ than at present. Note that an evolution of the GRB distributions with the redshift is not included in this procedure, although such evolution probably exists (e.g. Daigne et al. 2006). This case is not addressed here because we do not have a sufficient number of GRBs;

(3) the total smoothed distribution (SD) is the sum of the individual functions $f_{b}\left(l e_{\mathrm{p}, i}\right)$, normalized by the corresponding burst's weight $W_{b}$

$$
\mathrm{SD}_{\left(L E_{\text {peak,sample }}^{\text {intr }}\right)}=\frac{1}{n_{\text {simu }}} \times \sum_{b=1}^{n_{\text {burst }}} W_{b} \times f_{b}\left(l e_{\mathrm{p}, i}\right)
$$

with $n_{\text {simu }}$ the number of simulations (100) for each burst and $n_{\text {burst }}$ the number of bursts contained in our sample (82).

\subsubsection{Results}

The resulting unbiased $E_{\text {peak }}$ distribution for our sample of bursts with this method is shown in Fig. 6. In this figure we see that the $E_{\text {peak }}$ distribution has dramatically changed, with a clear domination of the bursts having low intrinsic $E_{\text {peak }}$, i.e. the X-ray flashes.

As explained in Sect. 3, we tested whether a high accuracy for the pseudo-redshifts was necessary, i.e. whether the results obtained could depend on these estimated distance scales. To do this, we performed the same analysis as described throughout this Section, but this time for simulated redshifts randomly selected within the error ranges based on the initial ones and artificially increased by factors of 1.4, 2.0 and 3.0. We find that both the distribution of intrinsic $E_{\text {peak }}$ and the unbiased distribution of intrinsic $E_{\text {peak }}$ do not significantly depend on the accuracy of the pseudo- $z$ within a factor 2 or 3 (Fig. 6). This result can be explained by the intrinsic dispersion of the parameters studied which is so large that the uncertainties in the distance scales do not strongly affect the results.

Moreover, we also performed this analysis by assuming for the distance of each of the 62 GRBs without secure redshift a 


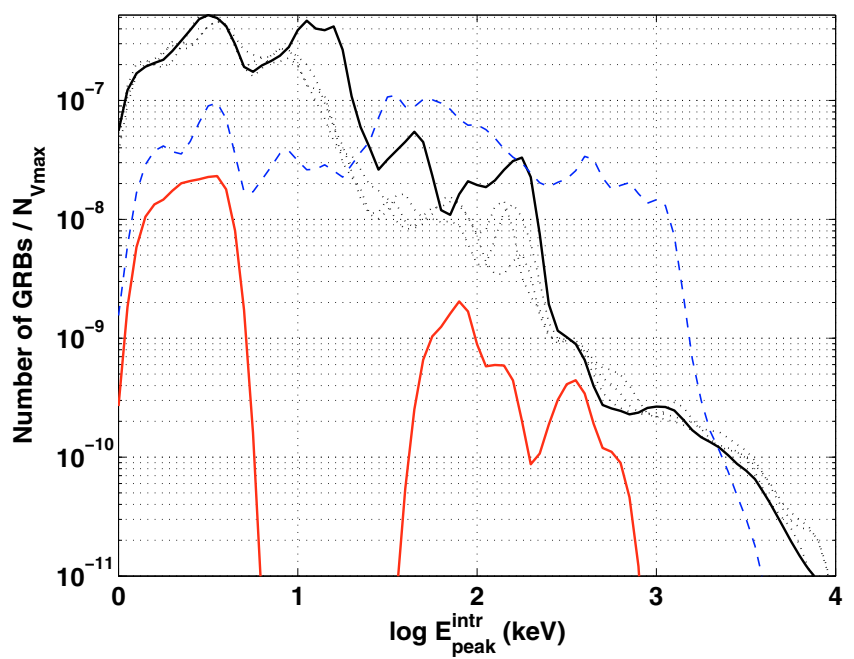

Fig. 6. Unbiased distributions of the intrinsic $E_{\text {peak }}$ for the complete sample of 82 GRBs detected and localized by HETE-2. The smoothed distribution (SD) based on the 20 bursts having a spectroscopic/photometric redshift $(z)$ is represented by the lower solid red curve and the one corresponding to the complete sample is shown with the upper solid black curve. The three black dotted smoothed distributions show the test performed to quantify the impact of the pseudoredshift accuracy in this study (all the pseudo-redshifts are given an error range artificially increased by factors of 1.4, 2.0 and 3.0). The blue dashed SD corresponds to case 2 (see Part 3.3), i.e. the sample of 6200 simulated GRBs (corresponding to the 62 GRBs of our sample without secure redshift) which are randomly given a redshift from the HETE-2 redshift distribution.

set of 100 redshifts randomly taken from the total redshift distribution of HETE-2 long bursts ( 24 values). The results obtained in this case differ significantly because the predominence of low $E_{\text {peak }}^{\text {intr }}$ bursts has disappeared. With this method, the distribution is flat and broad. Here again, the distribution's width may not reflect the true one, but it contributes interesting information to the unbiased $E_{\text {peak }}$ distribution, which is probably situated - "in reality" - between the two curves presented in Fig. 6 (black solid line and blue dashed line).

\section{Parameter 2: $T_{90}$}

In this section we study the duration distribution of the complete sample of HETE-2 GRBs. We adopted $T_{90}$ as the common definition for all the bursts. We derive in the following sub-sections the observed, intrinsic and unbiased $\log T_{90}$ distributions using the same method as described for the study of the intrinsic $\log E_{\text {peak }}$ distribution (Sect. 4).

\subsection{The observed $T_{90}$ distribution}

$T_{90}$ is defined as the duration for which $90 \%$ of the counts in a given energy range are detected. Recall that in this study we only focus on long-duration GRBs. Over the 4 FREGATE bands ( $A$ : 6-40 keV; B: 6-80 keV; $C$ : $30-400 \mathrm{keV} ; D$ : >400 keV) we chose the one that contained both the classical GRBs and the XRFs, i.e. the band $B(6-80 \mathrm{keV})$. The $\log T_{90}$ distribution in this energy range (see Fig. 7) extends from 0.33 to 2.73 . The mean value of this distribution is 1.40 and the median value 1.33.

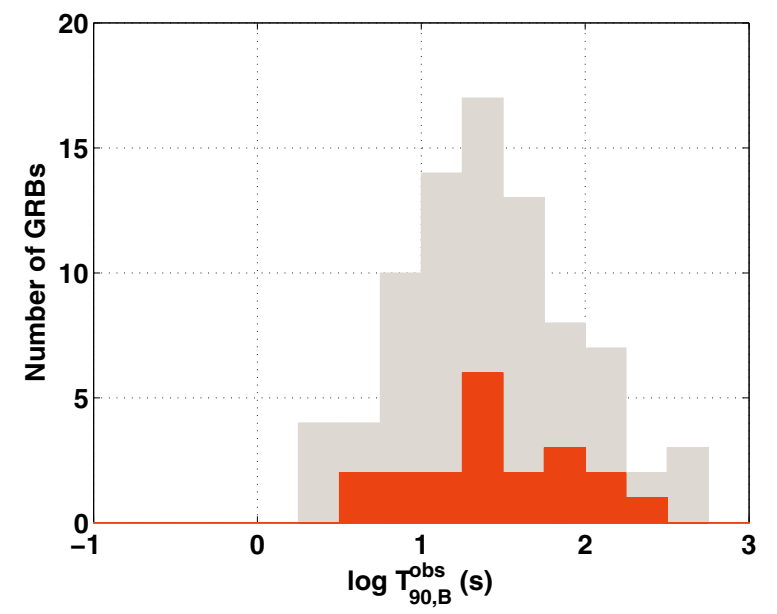

Fig. 7. Distributions of the $\log T_{90}$ measured in the observer frame and in band $B$ of FREGATE $(6-80 \mathrm{keV})$ for the complete sample of 82 GRBs localized by HETE-2. The histogram related to the subsample of bursts with secure redshifts $(z)$ is shown in red, and that of the total sample is shown in grey.

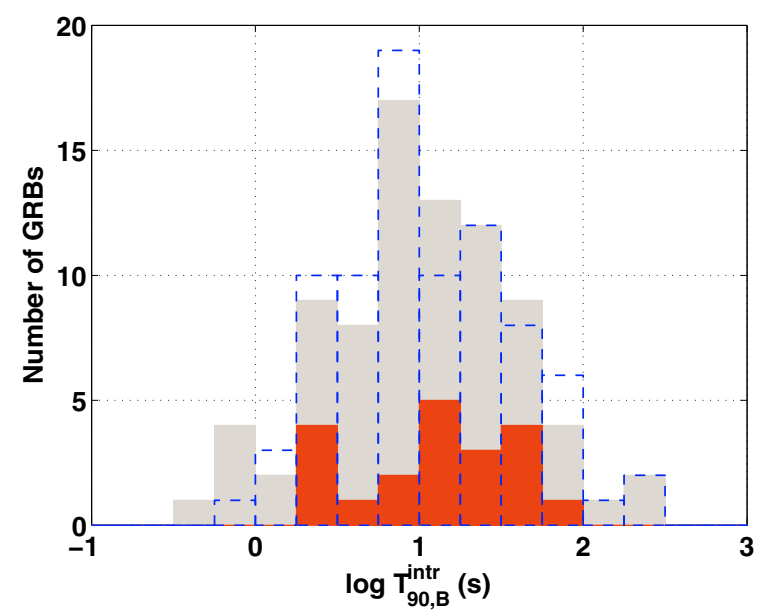

Fig. 8. Intrinsic $\log T_{90}$ distributions for the complete sample of 82 GRBs detected and localized by HETE-2. The histogram of the subsample of bursts with secure redshifts $(z)$ is shown in red, and the total sample histogram is shown in grey. The blue dashed histogram corresponds to the case 2 (see Part 3.3), i.e. to the 62 GRBs without secure redshift that were randomly given a redshift from the HETE-2 redshift distribution.

\subsection{The intrinsic $T_{90}$ distribution}

The histograms are shown in Fig. 8. We caution that the observed $T_{90}$ are measured in the same energy range in the observer frame (see Table 2). Since this energy range depends on the redshift of the source, it is different for each burst and this has an impact on the intrinsic duration measurement.

The histogram of the intrinsic $\log T_{90}$ in the $(6-80 \mathrm{keV})$ energy range extends from -0.26 to 2.40 . The mean value of this distribution is 1.01 and the median value is 1.00 .

Contrary to the $\log E_{\text {peak }}$ distribution, the intrinsic $\log T_{90}$ distribution is not broader than the observed $\log T_{90}$ distribution and is extended over about the same range ( 2.5 decades $)$. 


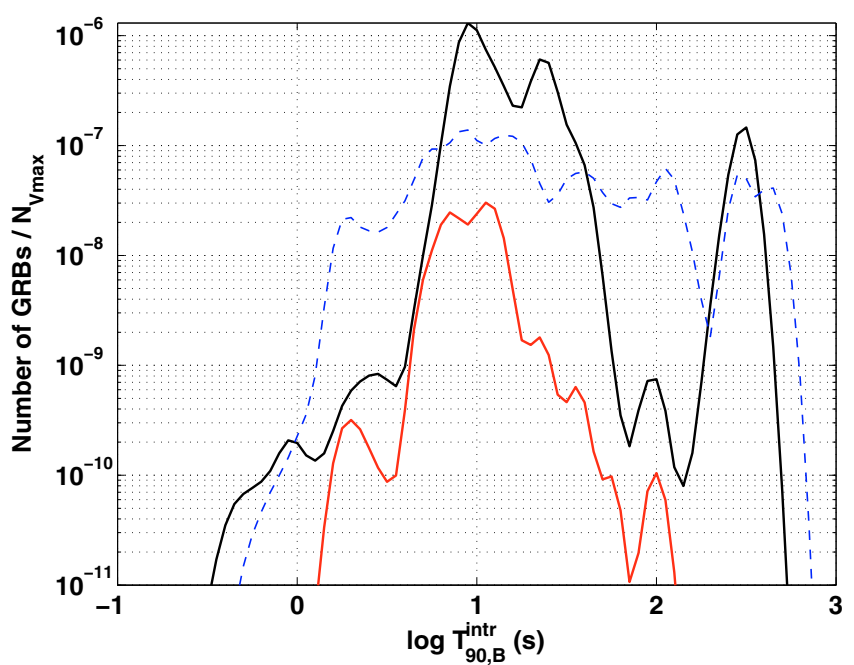

Fig. 9. Unbiased distributions of the intrinsic $\log T_{90}$ for the complete sample of 82 GRBs detected and localized by HETE-2. The smoothed distribution based on the 20 bursts with spectroscopic/photometric redshifts $(z)$ is represented by the lower solid red curve; the one for the complete sample is given by the upper solid black line. The blue dashed SD corresponds to case 2 (see Part 3.3), i.e. the sample of 6200 simulated GRBs (corresponding to the 62 GRBs of our sample without secure redshift) which were randomly given a redshift from the HETE-2 redshift distribution.

\subsection{The unbiased $T_{90}$ distribution}

Correcting the intrinsic $T_{90}$ for the $N_{V \max }$ (see Part 4.4) we obtained the unbiased $T_{90}$ distribution (Fig. 9).

We note that the GRB population is dominated by events with an intrinsic duration of about $10 \mathrm{~s}$ (for an energy range of $6-80 \mathrm{keV}$ in the observer frame). Nevertheless, a nonnegligable number of GRBs have a long intrinsic duration lasting about $300 \mathrm{~s}$.

If we consider the case 2 study (Fig. 9), we obtain a significantly different unbiased $T_{90}$ distribution, more flat than the one obtained in case 1, i.e. no typical intrinsic duration for the GRB appears when considering distances randomly taken within the total redshift distribution of HETE-2 GRBs.

\section{Parameter 3: isotropic energy}

\subsection{The observed fluence}

Taking the fluences (i.e. the fluxes integrated over the duration of the bursts' spectra) in the HETE-2 Catalog (Vanderspek et al. 2008) and applying the same method described in Sect. 4, we show in Fig. 10 the histograms of the logarithmic fluence in the energy range $2-30 \mathrm{keV}$. The distribution of the $\log S_{\mathrm{X}}$ extends between -7.12 and -4.24 . The mean and median values of this distribution are -5.90 and -6.00 , respectively.

\subsection{The isotropic energy}

The equivalent of the fluence measured in the observer frame is the energy radiated in the source frame. One easy way to estimate this energy is to assume an isotropic emission $\left(E_{\text {iso }}\right)$ integrated over a fixed energy-range $\left(1-10^{4} \mathrm{keV}\right.$ in the observer frame, see e.g. Amati et al. 2002; Amati 2006). We computed this quantity for the 82 bursts and obtained the distribution shown in Fig. 11. This distribution of $\log E_{\text {iso }}$ extends from 49.50 to 54.24 , and has a mean value of 52.44 and a median value of

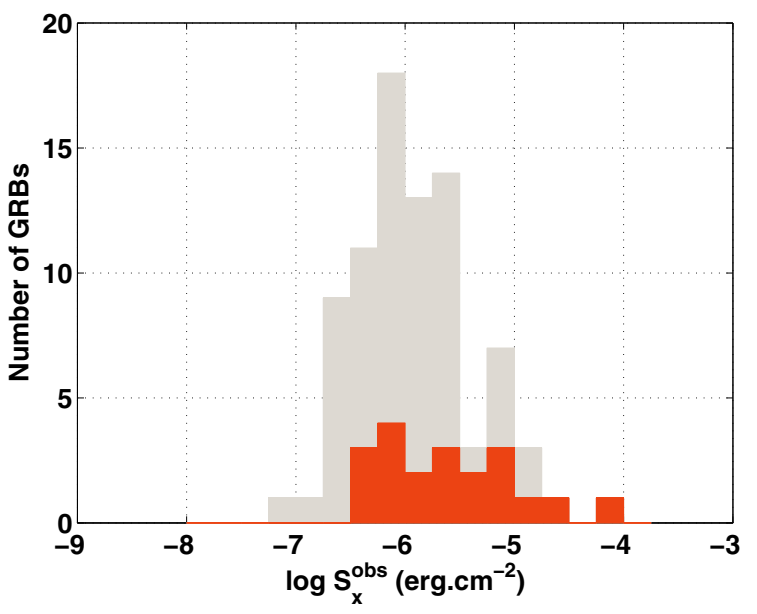

Fig. 10. Distributions of the logarithmic fluence in the $X$ energy range $(2-30 \mathrm{keV})$ for the complete sample of 82 GRBs localized by HETE-2. The histogram for the sub-sample of bursts with secure redshifts $(z)$ is shown in red, and the total sample histogram is shown in grey.

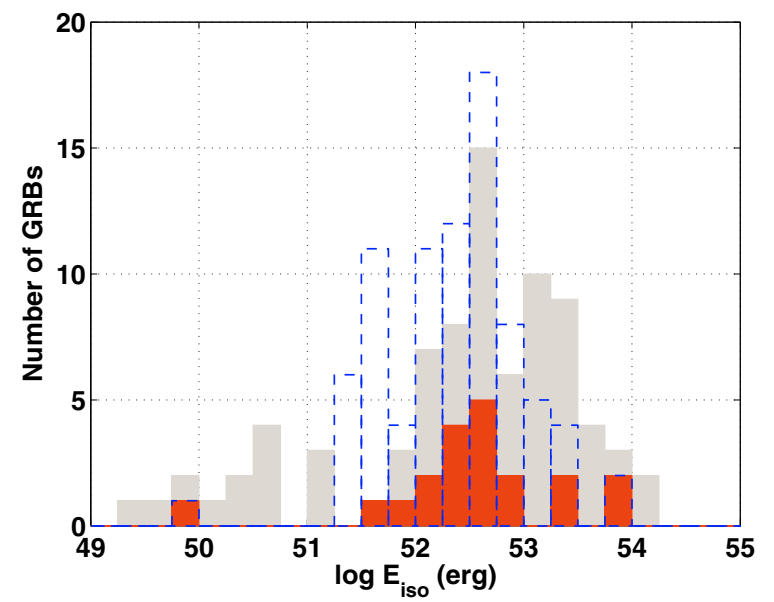

Fig. 11. $E_{\text {iso }}$ distributions for the complete sample of 82 GRBs localized by HETE-2. The histogram of the sub-sample of bursts with secure redshifts $(z)$ is shown in red, and the histogram of the total sample is shown in grey. The blue dashed histogram corresponds to case 2 (see Part 3.3), i.e. to the sample of 62 GRBs without secure redshifts that were randomly given a redshift from the HETE-2 redshift distribution.

52.62. Two classes of bursts are evident, with a separation at about 51.5. Most of the bursts have a $\log E_{\text {iso }}$ lying between 52 and 54 (the classical GRBs) and the second sample has a $\log E_{\text {iso }}$ lower than 51 .

\subsection{The unbiased isotropic energy}

The correction of these $E_{\text {iso }}$ for the $N_{V \max }$ of each burst gives us the unbiased $E_{\text {iso }}$ distribution (see Fig. 12). This distribution decreases with $E_{\text {iso }}$, and the best-fit power-law for the complete sample distribution, estimated between $E_{\text {iso }}=10^{50} \mathrm{erg}$ and $E_{\text {iso }}=$ $10^{54} \mathrm{erg}$ is $10^{-7.94} \times E_{\text {iso, } 52}^{-0.84}$, where $E_{\text {iso,52 }}$ is in units of $10^{52} \mathrm{erg}$. As for the two previous cases studied, this distribution is dramatically different from the observed and intrinsic ones. It shows a clear predominence of bursts with low $E_{\text {iso. This distribution }}$ hence strengthens the result we obtained in Sect. 4, i.e. the predominence of X-ray flashes in the overall GRB population. Here again we can discuss the impact of our second case calculation. 


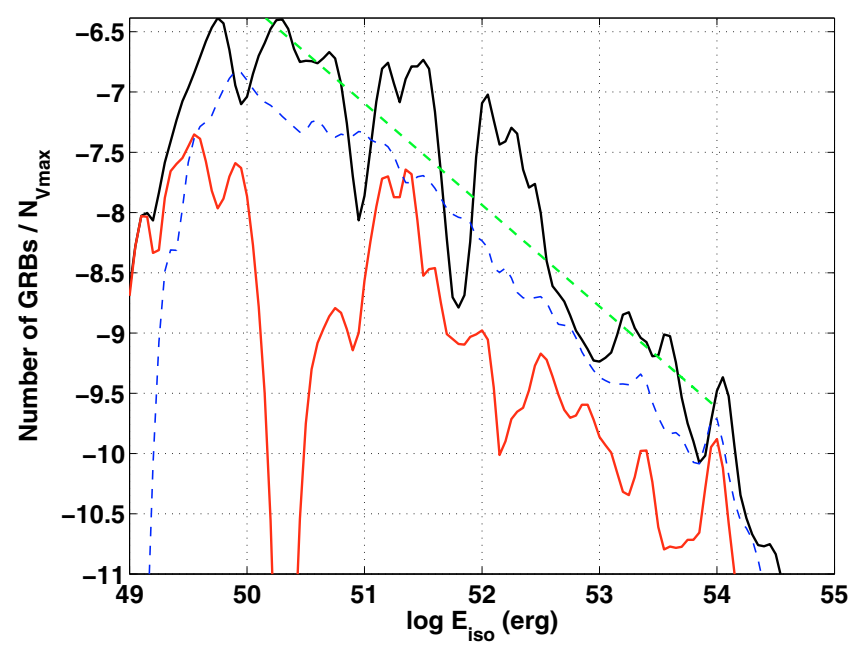

Fig. 12. Unbiased $E_{\text {iso }}$ distributions for the complete sample of $82 \mathrm{GRBs}$ localized by HETE-2. The smoothed distribution based on the 20 bursts with a spectroscopic/photometric redshift $(z)$ is represented by the lower solid red curve; the one for the complete sample is shown by the upper solid black line. The blue dashed SD corresponds to case 2 (see Part 3.3), i.e. the sample of 6200 simulated GRBs (corresponding to the 62 GRBs of our sample without secure redshifts) which were randomly given a redshift from the HETE-2 redshift distribution. The bestfit power-law (green dashed line) for the complete sample, estimated between $E_{\text {iso }}=10^{50}$ and $10^{54}$ erg is $10^{-7.94} \times\left(\frac{E_{\text {iso }}}{10^{52}}\right)^{-0.84}$.

Table 1. Correlation coefficients $(R)$ obtained for the pairwise comparison of the four intrinsic parameters discussed in this paper $\left(E_{\text {peak }}, T_{90}\right.$, $\left.E_{\text {iso }}, z_{\text {sample }}\right)$. The lower and upper bounds are at a $95 \%$ confidence interval. The corresponding $p$-values test the non-correlation by computing the probability of having a correlation as large as the observed value by chance.

\begin{tabular}{lll}
\hline \hline Tested correlation & $R$ & $p$-values \\
\hline$E_{\text {iso }}-E_{\text {peak }}^{\text {intr }}$ & $0.912_{-0.046}^{+0.031}$ & $1.215 \times 10^{-32}$ \\
$z_{\text {sample }}-E_{\text {peak }}^{\text {intr }}$ & $0.860_{-0.070}^{+0.048}$ & $4.297 \times 10^{-25}$ \\
$E_{\text {iso }}-T_{90}^{\text {int }}$ & $-0.102_{-0.211}^{+0.220}$ & $3.596 \times 10^{-1}$ \\
$z_{\text {sample }}-T_{90}^{\text {intr }}$ & $-0.354_{-0.177}^{+0.217}$ & $1.087 \times 10^{-3}$ \\
$T_{90}^{\text {intr }}-E_{\text {peak }}^{\text {int }}$ & $-0.255_{-0.193}^{+0.215}$ & $2.060 \times 10^{-2}$ \\
$z_{\text {sample }}-E_{\text {iso }}$ & $0.831_{-0.083}^{+0.057}$ & $4.729 \times 10^{-22}$ \\
\hline
\end{tabular}

In this case, the shape of the distribution is similar to the one obtained in case 1 .

\section{Correlations}

Having determined the intrinsic properties of the HETE-2 GRBs, we test whether any correlations are found between these values. We performed a pairwise comparison of the four intrinsic parameters ( $\left.E_{\text {peak }}, T_{90}, E_{\text {iso }}, z_{\text {sample }}\right)$ to search for possible correlations. Six plots are obtained and shown in Fig. 13. The corresponding correlation coefficients and associated $p$-values testing the hypothesis of no correlation are presented in Table 1 . We note that the $E_{\text {peak }}^{\text {intr }}-E_{\text {iso }}$ (Amati) correlation is found (1st panel) and that the sub-sample of XRFs fills the gap between XRF 020903 and the cluster of intrinsic classical GRBs (see next section). This is not really surprising since our pseudo-redshift estimates assume that the $E_{\text {peak }}^{\text {intr }}-E_{\text {iso }}$ relation is valid. It is nevertheless interesting to note that nearly all of the 82 GRBs in our sample are consistent with this correlation with no strong outlier.
While it is not the purpose of this paper to discuss the reality of $E_{\text {peak }}^{\text {intr }}-z_{\text {sample }}$ and $E_{\text {iso }}-z_{\text {sample }}$ correlations, we note that these correlations could be partially or totally explained by selection effects which practically prevent the detection of low-luminosity GRBs at high redshift. In the three last plots, no other "simple" tight correlation is found, but these plots interestingly show the instrumental limits affecting the detection of the bursts (duration, energetics and redshift). For instance, we can clearly see that HETE-2 was lucky to detect the bright GRB 030329 (see panels 2 and 6). Hence Fig. 13 can be used to show the characteristics of the bursts missed by HETE-2, i.e. mainly the GRBs at low-redshift with high $E_{\text {peak }}^{\text {intr }}$ or high $E_{\text {iso }}$; and conversely the GRBs with low $E_{\text {peak }}$ occuring at high redshift.

\section{The nature of X-ray flashes}

The demonstration of the existence of X-ray flashes (Heise et al. 2001; Kippen et al. 2001) was immediately followed by several theories attempting to explain their nature and to determine whether they belong to the same class as the classical GRBs, or whether they are different cosmological events. Thanks to various studies of samples of X-ray flashes, several clues now allow us to unambiguously associate XRFs with the classical GRBs. XRFs are GRBs with lower $E_{\text {peak }}$ and higher fluxes in $X$-rays than in $\gamma$-rays (e.g. Kippen et al. 2003, 2004; Barraud et al. 2003; Sakamoto et al. 2005). The other spectral parameters of the prompt emission and the temporal behaviour of XRF afterglows at various wavelengths are identical to those of classical GRBs (e.g. D'Alessio et al. 2006).

In our complete sample, we have 22 bursts classified as XRFs. In the following section we discuss some results obtained with this sample.

\subsection{The redshift distribution of HETE-2-XRFS}

To explain the origin of the XRFs, Heise et al. (2001) proposed that they are classical GRBs occuring at high redshift. If we consider the redshift distribution of the HETE-2 complete sample, we note that the sub-sample of XRFs has a mean distance scale $\left\langle z_{\text {sample }}\right\rangle_{\mathrm{XRFs}}^{\mathrm{HETE}-2}=0.82$, lower than the mean redshift of the "classical" GRBs: $\left\langle z_{\text {sample }}\right\rangle_{\text {GRBs }}^{\mathrm{HETE}-2}=2.09$ (see Fig. 14). The mean redshift of the XRFs contained in our sample is also lower than the mean redshift of the Swift XRFs derived by Gendre et al. (2007): $\langle z\rangle_{\mathrm{XRFs}}^{\mathrm{Swift}}=1.40$ for a sample of 9 XRFs with known redshifts. Consequently, we reject this hypothesis, as was previously done in other studies, e.g. by Barraud et al. (2003) who highlighted this fact with the similar duration distributions they obtained for the XRFs and the long classical GRBs.

\subsection{Do X-ray flashes form a distinct population?}

We consider in this paragraph the question posed, e.g., in Stratta et al. (2007): do the XRFs form a continuum extending the "classical GRBs" to soft energies, or do they form a distinct population, the intrinsic XRFs $(i-X R F s)$ ?

Figure 13 (panel 1) shows the Amati relation for the complete sample of 82 HETE-2 bursts and Fig. 13 (panel 2) plots the $E_{\text {peak }}^{\text {intr }}$ versus the distance-scales of the sources. We do not find any clear evidence for a distinct population and the results indicate a continuum between the classical GRBs and the XRFs. Indeed, according to the HETE-2 sample, several XRFs are situated in the gap between the group of intrinsic classical GRBs and the two intrinsic XRFs 020903 (Sakamoto et al. 2004) and 

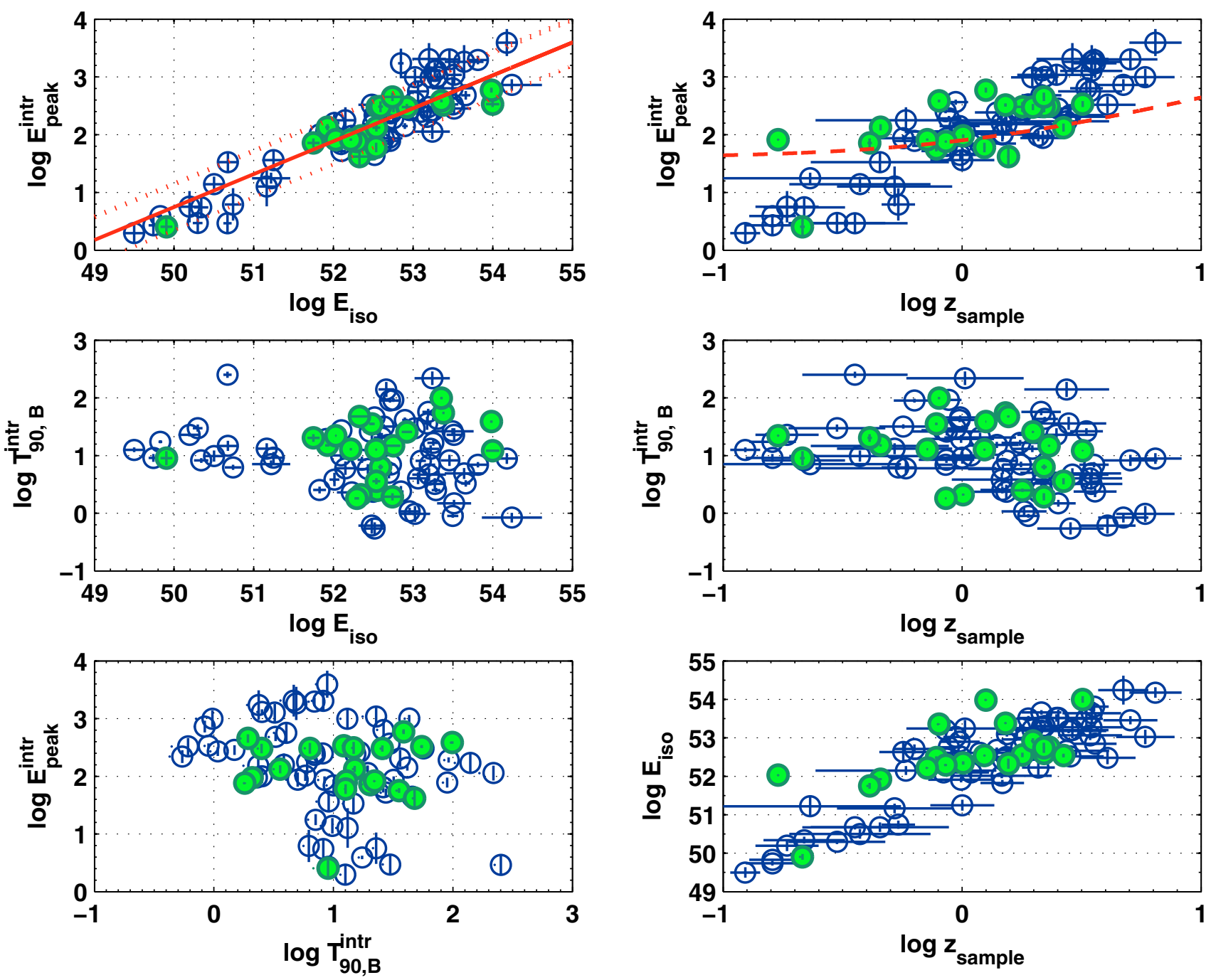

Fig. 13. Correlations between the intrinsic parameters studied: $E_{\text {peak }}^{\text {intr }}, T_{90}^{\text {intr }}, E_{\text {iso }}$ and the distance-scale $z_{\text {sample. }}$. In each panel the GRBs that have a measured redshift $(z)$ are represented with the green filled circles, and the ones having a pseudo-redshift $(\hat{z})$ are symbolized with the blue open circles. In the top left panel, by way of comparison, we show (solid line) the best-fit power-law $E_{\text {peak }}^{\text {intr }}=77 \times{ }_{\text {iso, } 52}^{0.57}\left(\right.$ with $E_{\text {peak }}^{\text {intr }}$ in keV and $E_{\text {iso, }, 52}$ in units of $10^{52} \mathrm{erg}$ ) found by Amati (2006) and also the vertical logarithmic deviation of 0.4 (dotted lines) displayed in Amati (2006). In the top right panel the dashed curve marks the redshift evolution of the $E_{\text {peak }}$, assuming a burst with $E_{\text {peak }}^{(z=0)}=40 \mathrm{keV}$ (see Stratta et al. 2007).

060218 (Amati et al. 2007), as is XRF 050416, a soft burst detected by Swift (Sakamoto et al. 2006a,b). However, half of the sub-sample of the observed XRFs are part of the cluster of intrinsic classical GRBs when considered in their source frame. This fact was also noted by Stratta et al. (2007).

\section{The rate of GRBs in the Local Universe}

\subsection{The rate of GRBs in the Local Universe, as measured by HETE-2}

The various corrections (distance scale, number of GRB within the visibility volume) applied to the distributions obtained in the observer frame allowed us to obtain the unbiased distributions of the intrinsic parameters, i.e. distributions that reflect more closely the true distributions of these parameters and the dominant characteristics of the gamma-ray burst population.

This study also allows us to derive the rate of GRBs detected and localized by HETE- $2\left(R_{0}^{\mathrm{H} 2}\right)$. For that purpose, we consider that each GRB in our sample contributes to the local rate in proportion to:

$h_{b}=\frac{N_{V l o c}(z=0.1)}{N_{V \max }\left(z=z_{b, \max }\right)}$

with the $N_{V}(z)$ computed according to Eq. (3), and we obtain the rate of HETE-2 GRBs during the mission,

$\tau=\frac{1}{V_{\mathrm{loc}}} \sum_{b=1}^{n_{\text {burst }}} h_{b}$

which is $3.30 \mathrm{Gpc}^{-3}$.

In order to normalize this rate per year, we took into account the effective monitoring time of the WXM, obtained from:

$T_{\mathrm{m}}=\frac{T_{\text {mission }} \times T_{\epsilon}}{4 \pi} \times S_{\mathrm{cov}}$

where $T_{\mathrm{m}}$ is the effective monitoring time of the $\mathrm{WXM}, T_{\text {mission }}=$ 69 months is the duration of the mission, $T_{\epsilon}=37 \%$ is the 


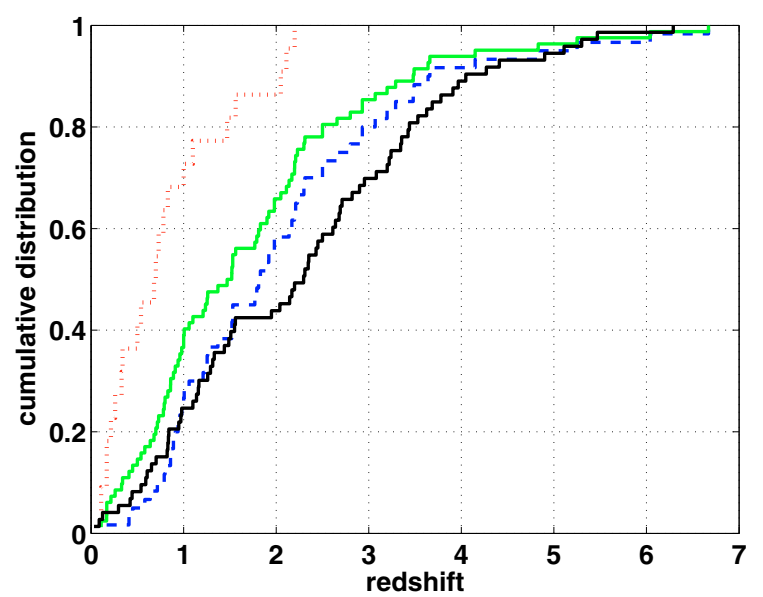

Fig. 14. Four distance-scale cumulative distribution functions (cdf). The cdf of the HETE-2 complete sample is shown with the green solid line. The two cumulative distributions for the "classical" GRBs and the XRFs are shown with the blue dashed line and the red dotted line, respectively. We note that most of the $22 \mathrm{XRFs}$ are definitely situated at intermediate or low redshift. For comparison, we also considered all the long-duration bursts with redshifts detected by Swift-BAT from the beginning of the mission to GRB 080210 (the last Swift burst with a spectroscopic redshift measured to date). The corresponding cdf is the black solid line.

mean observation efficiency during $T_{\text {mission }}$, and $S_{\text {cov }}=2 \pi(1-$ $\left.\cos 45^{\circ}\right)=1.84$ sr the sky-coverage of the $\mathrm{WXM}^{8}$.

The effective monitoring time of the WXM is hence $T_{\mathrm{m}}=$ $0.31 \mathrm{yr}$. Using this, the rate of GRBs in the Local Universe per $\mathrm{Gpc}^{3}$ and per year can be found to be $\sim 10.6 \mathrm{Gpc}^{-3} \mathrm{yr}^{-1}$. This result is a lower limit because as shown in Sect. 4, the bursts that dominate the overall population are the ones with low $E_{\text {peak }}^{\text {intr }}$, and as we showed in Sect. 7, HETE-2 missed the bursts with intrinsic $E_{\text {peak }}$ lower than 1 or $2 \mathrm{keV}$ as well as bursts occuring at very high redshifts.

If we consider the unbiased distributions obtained in case 2 (shown as dotted blue lines throughout the different figures), we obtain $1.02 \mathrm{Gpc}^{-3}$, corresponding to a rate of $\sim 3.3 \mathrm{Gpc}^{-3} \mathrm{yr}^{-1}$.

As shown in Sect. 4, the population of gamma-ray bursts is dominated by the X-ray flashes. This is understandable as XRFs are soft but also faint in the observer frame, according to the hardness-intensity relation derived by Barraud et al. (2003). Therefore, if the rate of detected bursts is in fact higher for classical GRBs than for XRFs, we can guess that this is only due to instrumental limitations, such as the size and the sensitivity of the detectors.

\subsection{Comparison with the rates of GRBs obtained in previous studies}

Several studies have derived local rates of GRBs using various methods mainly based on theoretical considerations.

According to the results obtained so far, the predicted rate of GRBs in the Universe seems to be between unity (or less) GRB and several hundreds per $\mathrm{Gpc}^{3}$ and per year. These results seem to be related to the nature of the bursts considered in the studies, i.e. (1) "classical GRBs" also called "high-luminosity GRBs" (HL-GRBs) and/or (2) sub-luminous (low-luminosity) GRBs (LL-GRBs).

\footnotetext{
${ }^{8}$ Recall that throughout this study, we only consider the GRBs localized by the WXM.
}

(1) Several authors obtained a GRB rate close to $1 \mathrm{Gpc}^{-3} \mathrm{yr}^{-1}$. The method they used is generally based on the determination of the luminosity function (LF) by fitting a phenomenological model to the $\log (N)-\log (P)$ relation of wellknown GRB catalogs. Assuming that GRBs trace the star formation rate (SFR), a model of the SFR is adopted for it and the rate of GRBs is determined.

For instance, Guetta et al. $(2004,2005)$ use the 2204 GRBs of the GUSBAD Catalog (Schmidt 2004) and a sample consisting of 595 long GRBs detected by BATSE. Following Schmidt (2001), they use these samples to find the luminosity function by fitting a single power-law model to the BATSE observed peak flux distribution. In addition, they assumed that the GRB rate follows the SFR and hence adopted either the SFR model $\mathrm{SFR}_{2}$ of Porciani \& Madau (2001) or the SFR model of Rowan-Robinson (1999). The estimated rate lies between 0.1 and $1.1 \mathrm{Gpc}^{-3} \mathrm{yr}^{-1}$. This rate is in agreement with the one found by Schmidt (2001): $0.5 \mathrm{Gpc}^{-3} \mathrm{yr}^{-1}$ with $1391 \mathrm{GRBs}$ contained in the BATSE DISCLA 2 Catalog (Schmidt 1999) and the one recently found by Liang et al. (2007) $\left(1.12_{-0.20}^{+0.43} \mathrm{Gpc}^{-3} \mathrm{yr}^{-1}\right)$ with a sample of $45 \mathrm{HL}$ Swift-GRBs. The rate of HL HETE-2 GRBs is also consistent with all these results, since we obtain a lower limit $R_{0}^{\mathrm{H} 2} \gtrsim 0.45 \mathrm{Gpc}^{-3} \mathrm{yr}^{-1}$ (see Part 9.3).

(2) GRB 060218, detected by Swift-BAT (Cusumano et al. 2006), was found to be at very low-redshift $z=0.0331$ (Mirabal et al. 2006a). Moreover, its association with the supernova SN 2006aj (Pian et al. 2006) recalled the characteristics of GRB 980425, the closest GRB ever identified, at a redshift $z=0.0085$ (Tinney et al. 1998). These GRBs associated with $\mathrm{SNe}$ are peculiar in the sense that they constitute the two nearest long duration bursts detected in eight years. Liang et al. (2007) show that the detection of these two events implies a local rate of low-luminosity GRBs (LL-GRBs) of $R_{\mathrm{LL}} \sim 800 \mathrm{Gpc}^{-3} \mathrm{yr}^{-1}$, in agreement with Guetta \& Della Valle (2007) who find a rate of GRB 980425-like events of $\sim 430 \mathrm{Gpc}^{-3} \mathrm{yr}^{-1}$ and a rate of GRB 060218-like events of $\sim 350 \mathrm{Gpc}^{-3} \mathrm{yr}^{-1}$.

The rate of LL-GRBs, which is considerably higher than the rate of HL-GRBs, could be explained in two ways: first, the $\log (N)-\log (P)$ distribution of BATSE bursts often used to fit the GRB luminosity function cannot constrain it down to such low luminosities, because a GRB 060218-like event could not trigger BATSE. Second, the use of a phenomenological model for the GRB LF requires a choice for the extrema values $L_{\min }$ and $L_{\max }$. However, due to instrumental limitations, the true $L_{\min }$ of GRBs is unknown. Thus, the number of low-luminosity GRB is quite uncertain.

\subsection{Discussion}

We showed that the study of the complete sample of GRBs localized by the WXM on-board HETE- 2 led to a rate $R_{0}^{\mathrm{H} 2} \gtrsim$ $10.6 \mathrm{Gpc}^{-3} \mathrm{yr}^{-1}$. Our result is independent of any assumption on the luminosity function, as it is based on measured data. We note that this measured rate is at least 10 times higher than the HL-GRB rate and 10 times lower than the LL-GRB one. How can we explain this "intermediate" result?

- $\left(R_{0}^{\mathrm{H} 2}<R_{0}^{\mathrm{LL}}\right)$ : we first note that we have no event like GRB 980425 or GRB 060218 in our sample. Previous studies showed that it was impossible for an HETE-like mission with a WXM-like camera to detect a burst like XRF 060218. This burst occured at a distance $d=145 \mathrm{Mpc}$, and Soderberg et al. (2006c) show that the WXM could have detected it at a maximum distance of $110 \mathrm{Mpc}$. For a burst similar to 
GRB 980425 occurring at $d=36.1 \mathrm{Mpc}$, the WXM could have detected it to a distance $d=60 \mathrm{Mpc}$. However, Guetta et al. (2004) show that with their model of the LF, the local rate of nearby bright bursts observable by HETE-2 within this distance is only $0.057 \mathrm{yr}^{-1}$.

- $\left(R_{0}^{\mathrm{H} 2}>R_{0}^{\mathrm{HL}}\right)$ : if we now imagine that HETE-2 was a mission that could not detect X-ray flashes, we can try to re-do all the steps to derive the rate of GRBs detected and localized by it (see Part 9.1) in the sample of 60 "classical" GRBs. We obtain $R_{0}^{\mathrm{H} 2} \gtrsim 0.45 \mathrm{Gpc}^{-3} \mathrm{yr}^{-1}$. This result is in good agreement with the rates usually obtained when only the HL-GRBS are considered: [0.1-1] $\mathrm{Gpc}^{-3} \mathrm{yr}^{-1}$.

Consequently, we infer that the difference between the rate of HL-GRBs obtained with BATSE and the rate measured with HETE-2 is due to the X-ray flashes. These soft events cannot be detected in the BATSE energy range (50-300 keV). With our method based on observed properties of GRBs, XRFs and classical GRBs can be distinguished. The average lower redshift of XRFs leads to higher weights for the XRFs than for classical $\mathrm{GRBs}^{9}$. In fact, it appears that XRFs constitute an intermediate class in terms of frequency between the HL-GRBs and the LL-GRBs, and the rate of XRFs could be higher than the rate of classical GRBs by a factor of $\sim 10-100$.

Despite their higher softness, faintness and frequency, our study brings the XRFs closer to the classical GRBs. This is not the case for GRB 980425 or XRF 060218-like events that are undetectable and out of reach of a HETE-2-like mission.

\subsection{Comparison with the rate of type $\mathrm{lb} / \mathrm{c} S \mathrm{Ne}$}

If we compare the measured rate of GRBs based on the complete HETE-2 sample and the rate of $\mathrm{SNe} \mathrm{Ib} / \mathrm{c}$ considered in Soderberg et al. (2006c), $R_{0}^{\mathrm{SNeIb} / \mathrm{c}}=9_{-5}^{+3} \times 10^{3} \mathrm{Gpc}^{-3} \mathrm{yr}^{-1}$ measured by Cappellaro et al. (1999) and Dahlen et al. (2004), the ratio is $\gtrsim 0.1 \%$.

This low ratio highlights the fact that not all the type $\mathrm{Ib} / \mathrm{c} \mathrm{SNe}$ produce GRBs. The main explanation invokes a mildly relativistic jet, which is the source of the GRB, but that does not appear in all $\mathrm{SNe} \mathrm{Ib} / \mathrm{c}$. Indeed, if the optical luminosities of GRBs and $\mathrm{SNe}$ can be considered to be similar, optical spectroscopy reveals broad absorption lines in SNe spectra associated with GRBs, indicative of fast ejecta. These peculiar highly energetic $\mathrm{SNe}$, often designed Hypernovae ( $\mathrm{HNe}$ ) would not represent more than $3 \%$ of the $\mathrm{SNe} \mathrm{Ib} / \mathrm{c}$, as obtained by the studies based on the comparison of radio luminosities of GRB afterglows and $\mathrm{SNe} \mathrm{Ib} / \mathrm{c}$ (see e.g. Berger et al. 2003; Soderberg et al. 2006b; Soderberg 2007).

From previous studies of the predicted local rate of GRBs, and with our measured local rate, we now have a ratio between the $\mathrm{SNe} \mathrm{Ib} / \mathrm{c}$ and the GRBs lying in the range $\sim[0.1-3 \%]$. This small ratio could imply that the escape of the ultra-relativistic jet from the progenitor star is not the only ingredient needed to produce a GRB. In addition, several studies of stellar rotation, binarity, asymmetry and metallicity have shown that the progenitors must have some special characteristics in order to produce a gamma-ray burst.

\section{Summary}

In this paper we have presented the study of a sample containing all the gamma-ray bursts detected by HETE- 2 above a certain

\footnotetext{
9 Recall that the weight taken for each burst is inversely proportional to the number of GRBs within its visibility volume and is obviously redshift-dependent.
}

threshold and localized by the WXM. Our main goal was (1) to provide intrinsic distributions of the main global properties associated with the GRBs, i.e. taking into account the cosmological effects. The parameters studied are the peak energy $\left(E_{\text {peak }}\right)$, the duration $\left(T_{90}\right)$ and the total energy $\left(E_{\text {iso }}\right)$. The second purpose of this paper is (2) to derive unbiased distributions of these properties, taking into account the spatial density of GRBs, and to measure the rate of HETE-2 GRBs in the local universe.

(1) The main conclusions are the following:

- the intrinsic $E_{\text {peak }}$ distribution is quite broad, containing at the extreme sides of the energy range a large sample of X-ray flashes and few "classical" hard bursts. Nevertheless, we caution that the true width of this distribution is probably even broader than derived here, since the detector characteristics prevented HETE-2 from detecting bursts with an intrinsic peak energy of 1 or $2 \mathrm{keV}$ or, at the opposite end, bursts with an intrinsic peak energy of a few MeV;

- the intrinsic $T_{90}$ distribution shows that HETE-2 did not localize very long duration bursts $\left(T_{90}>1000 \mathrm{~s}\right)$. This may be a limitation of the orbit because due to its antisolar position, the detectors are turned off every half-orbit when they have the Earth in their field of view, i.e. every $\sim 45 \mathrm{~min}$. On the other hand, some events have an intrinsic duration below $2 \mathrm{~s}$ and might be considered as "intrinsically short";

- the intrinsic $E_{\text {iso }}$ distribution extends over more than four decades in energy. Most of the observed bursts have $E_{\text {iso }}$ higher than $10^{52}$ erg: the "classical" GRBs. The distribution extends down to lower $E_{\text {iso }}\left(10^{50}-10^{51} \mathrm{erg}\right)$, corresponding to the sample of HETE-2 XRFs.

\section{(2) The main conclusions are the following:}

- the "unbiased" distributions are dramatically different from their uncorrected shapes. Indeed, the unbiased $E_{\text {peak }}$ distribution shows a predominence of bursts with intrinsic $E_{\text {peak }}$ lower than $10 \mathrm{keV}$ and the unbiased $E_{\text {iso }}$ distribution shows a predominence of bursts with $E_{\text {iso }}$ lower than $10^{50}-10^{51} \mathrm{erg}$. The $E_{\text {iso }}$ distribution can be fitted between $10^{50}$ and $10^{54} \mathrm{erg}$ by a simple power-law with a slope of -0.84 .

However, we caution that the "simple" distributions of the intrinsic parameters probably suffer from biases. The same point may be made for the shapes of the "unbiased" distributions: the true ones are probably also different, as shown by the two studies performed using different distances for the bursts without secure redshifts - case 1: pseudo-redshifts and case 2: known spectroscopic redshifts randomly attributed which lead to different shapes for the $E_{\text {peak }}$ and the $T_{90}$ distributions. This emphasizes the predominence of X-ray flashes and the existence of a typical intrinsic duration for the GRBs in the first case, whereas a nearly constant number of GRBs through both the $E_{\text {peak }}$ energy range and the durations is highlighted in the second case. We may guess that the true distributions are situated between the results of these two cases;

- a possible application of our study is the determination of the rate of GRBs measured by HETE-2 in the Local Universe. We obtained a lower limit of $10.6 \mathrm{Gpc}^{-3} \mathrm{yr}^{-1}$, which is intermediate in magnitude between the rates usually found when a population of High-Luminosity GRBs is considered $\left(\sim 1 \mathrm{Gpc}^{-3} \mathrm{yr}^{-1}\right)$ or when the Low-Luminosity GRBs are also taken into account $\left(\gtrsim 100 \mathrm{Gpc}^{-3} \mathrm{yr}^{-1}\right)$. We explain this result with two arguments. First, HETE-2 did not detect LL-GRBs like GRB 980425 or XRF 060218 - mainly due to the small size of its detectors - so the rate cannot be as high as the one 
found for LL-GRBs. Second, it is the sample of XRFs detected by HETE-2 that makes the difference between the rate we obtained and the one found for HL-GRBs. As a consequence, thanks to the construction of unbiased distributions of the intrinsic properties of HETE-2 GRBS, we experimentally showed in this paper the dominance in terms of frequency of the X-ray flashes over the "classical GRBs", a frequency which is nevertheless lower than the sub-luminous bursts, and much smaller than the type $\mathrm{Ib} / \mathrm{c}$ supernovae.

Acknowledgements. The HETE-2 mission was supported in the US by NASA contract NASW-4690, in France by CNES contract 793-01-8479 and in Japan in part by the Ministry of Education, Culture, Sports, Science and Technology and by Grant-in-Aid for Scientific Research on Priority Areas 19047001. The authors acknowledge the valuable support of the HETE-2 Operation Team. AP is supported in France by the Ministry of National Education, Research and Technology. Y.E.N. is supported by the JSPS Research Fellowships for Young Scientists. This work is supported in part by a special postdoctoral researchers program in RIKEN. K.H. is grateful for support under MIT Contract SC-R293291. G. Pizzichini aknowledges financial support as part of ASI contract I/088/06/0. Finally, the authors acknowledge the referee for his/her valuable and relevant remarks that helped improving the content of this paper.

\section{References}

Amati, L. 2003, Chinese J. Astron. Astrophys. Suppl., 3, 455 Amati, L. 2006, MNRAS, 372, 233

Amati, L., Frontera, F., Tavani, M., et al. 2002, A\&A, 390, 81 Amati, L., Della Valle, M., Frontera, F., et al. 2007, A\&A, 463, 913 Ando, M., Ohta, K., Watanabe, C., et al. 2003a, GCN Circ., 1882 Ando, M., Ohta, K., Watanabe, C., et al. 2003b, GCN Circ., 1884 Arnaud, K., \& Dorman, B. 2003, XSPEC, An X-Ray Spectral Fitting Package, User's Guide for version $11.3 \mathrm{x}$

Atteia, J.-L. 2000, A\&A, 353, L18

Atteia, J.-L. 2003, A\&A, 407, L1

Atteia, J.-L., \& Pélangeon, A. 2005a, GCN Circ., 3476

Atteia, J.-L., \& Pélangeon, A. 2005b, GCN Circ., 3721

Atteia, J.-L., Boer, M., Cotin, F., et al. 2003, in Proc. of Gamma-Ray Burst and Afterglow Astronomy 2001, Woods Hole: A Workshop Celebrating the First Year of the HETE Mission, AIP Conf. Proc., 662, 17

Atteia, J.-L., Kawai, N., Vanderspek, R., et al. 2005, ApJ, 626, 292

Band, D., Matteson, J., Ford, L., et al. 1993, ApJ, 413, 281

Band, D., Cummings, J., Perri, M., et al. 2005, GCN Circ., 3466

Barraud, C., Olive, J.-F., Lestrade, J. P., et al. 2003, A\&A, 400, 1021

Barraud, C., Atteia, J.-L., Olive, J.-F., et al. 2004, AIPC, 727, 81

Barth, A. J., Sari, R., Cohen, M. H., et al. 2003, ApJ, 584, L47

Barthelmy, S. D., Cline, T. L., Butterworth, P., et al. 2000, in Proc. of Gamma-ray bursts: 5th Huntsville Symposium, AIP Conf. Proc., 526, 731

Berger, E., Kulkarni, S. R., Frail, D. A., \& Soderberg, A. M. 2003, ApJ, 599, 408 Berger, E., Gladders, M., \& Oemler, G. 2005, GCN Circ., 3201

Berger, E., Fox, B. B., Kulkarni, S. R., Frail, D. A., \& Djorgovski, S. G. 2007, ApJ, 660, 504

Bloom, J. S., Morrell, N., \& Mohanty, S. 2003, GCN Circ., 2212

Butler, N. R., Kocevski, D., Bloom, J. S., \& Curtis, J. L. 2007, ApJ, 671, 656

Caldwell, N., Garnavich, P., Holland, S., Matheson, T., \& Stanek, K. Z. 2003, GCN Circ., 2053

Campana, S., Mangano, V., Blustin, A. J., et al. 2006, Nature, 442, 1008

Cappellaro, E., Evans, R., \& Turatto, M. 1999, A\&A, 351, 459

Castro-Tirado, A. J., Pérez, E., Gorosabel, J., et al. 2002, GCN Circ., 1635

Cenko, S. B., Fox, D. B., Penprase, B. E., et al. 2008, ApJ, 677, 441

Cobb, B. E., Bailyn, C. D., van Dokkum, P. G., \& Natarajan, P. 2006, ApJ, 645, L113

Crew, G., Villasenor, J., Vanderspek, R., et al. 2002, GCN Circ., 1734

Crew, G. B., Lamb, D. Q., Ricker, G. R., et al. 2003, ApJ, 599, 387

Cusumano, G., Barthelmy, S., Gehrels, N., et al. 2006, GCN Circ., 4775

Dahlen, T., Strolger, L.-G., Riess, A. G., et al. 2004, ApJ, 613, 189

Daigne, F., Rossi, E. M., \& Mochkovitch, R. 2006, MNRAS, 372, 1034

D’Alessio, V., Piro, L., \& Rossi, E. M. 2006, A\&A, 460, 653

Della Valle, M. 2005, Interacting Binaries: Accretion, Evolution, and Outcomes, 797, 150

Della Valle, M. 2006a, in Proc. of Gamma-ray bursts in the Swift era: Sixteenth Maryland Astrophysics Conference, ed. S. S. Holt, N. Gehrels, \& J. Nousek, AIP Conf. Proc., 836, 367

Della Valle, M. 2006b, Chinese J. Astron. Astrophys. Suppl., 6, 315

Della Valle, M., Malesani, D., Benetti, S., et al. 2003, A\&A, 406, L33
Della Valle, M., Malesani, D., Bloom, J. S., et al. 2006a, ApJ, 642, 103 Della Valle, M., Chincarini, G., Panagia, N., et al. 2006b, Nature, 444, 1050 Djorgovski 2001, GCN Circ., 1108

Fenimore, E. E., \& Ramirez-Ruiz, E. 2000 [arXiv:astro-ph/0004176] Firmani, C., Ghisellini, G., Avila-Reese, V., \& Ghirlanda, G. 2006, MNRAS, 370,185

Foley, R. J., Chen, H.-W., Bloom, J., \& Prochaska, J. X. 2005, GCN Circ., 3483 Fox, D. B., Price, P. A., Soderberg, A. M., et al. 2003, ApJ, 586, L5

Fox, D. B., Berger, E., Price, P. A., et al. 2007, GCN Circ., 6071 Fugazza, D., Fiore, F., Cocchia, M., et al. 2003, GCN Circ., 1983 Fugazza, D., Fiore, F., Covino, S., et al. 2004, GCN Circ., 2782

Fynbo, J. P. U., Watson, D., Thöne, C. C., et al. 2006b, Nature, 444, 1047 Galama, T. J., Vreeswijk, P. M., van Paradijs, J., et al. 1998, Nature, 395, 670 Gal-Yam, A., Berger, E., Fox, D. B., et al. 2005, GCN Circ., 4156 Gal-Yam, A., Fox, D. B., Price, P. A., et al. 2006, Nature, 444, 1053 Gehrels, N., Chincarini, G., Giommi, P., et al. 2004, ApJ, 611, 1005 Gehrels, N., Norris, J. P., Barthelmy, S. D., et al. 2006, Nature, 444, 1044 Gendre, B., Galli, A., \& Piro, L. 2007, A\&A, 465, L13 Ghirlanda, G., Ghisellini, G., \& Lazzati, D. 2004, ApJ, 616, 331 Ghirlanda, G., Ghisellini, G., \& Firmani, C. 2006, New J. Phys., 8, 123 Golenetskii, S., Aptekar, R., Mazets, E., et al. 2005a, GCN Circ., 3474 Golenetskii, S., Aptekar, R., Mazets, E., et al. 2005b, GCN Circ., 3660 Götz, D., Mereghetti, S., Beck, M., et al. 2003, GCN Circ., 2459 Greiner, J., Guenther, E., Klose, S., et al. 2003a, GCN Circ., 1886 Greiner, J., Peimbert, M., Estaban, C., et al. 2003b, GCN Circ., 2020 Guetta, D., \& Della Valle, M. 2007, ApJ, 657, L73

Guetta, D., Perna, R., Stella, L., \& Vietri, M. 2004, ApJ, 615, L73 Guetta, D., Piran, T., \& Waxman, E. 2005, ApJ, 619, 412

Heise, J., in't Zand, J., Kippen, R. M., \& Woods, P. M. 2001, in Proc. 2nd Rome Workshop: Gamma-Ray Bursts in the Afterglow Era, ed. E. Costa, F. Frontera, \& J. Hjorth (Berlin: Springer-Verlag), 16

Hjorth, J., Sollerman, J., Møller, P., et al. 2003a, Nature, 423, 847 Hjorth, J., Møller, P., Gorosabel, J., et al. 2003b, ApJ, 597, 699 Jakobsson, P., Hjorth, J., Fynbo, J. P. U., et al. 2004, A\&A, 427, 785 Jakobsson, P., Frail, D. A., Fox, D. B., et al. 2005a, ApJ, 629, 45 Jakobsson, P., Fynbo, J. P. U., Paraficz, D., et al. 2005b, GCN Circ., 4029 Kaneko, Y., Preece, R. D., Briggs, M. S., et al. 2006, ApJ, 166, 298 Kelson, D. D., Koviak, K., Berger, E., et al. 2004, GCN Circ., 2627 Kippen, R. M., et al. 2001, in Proc. 2nd Rome Workshop: Gamma-Ray Bursts in the Afterglow Era, ed. E. Costa, F. Frontera, \& J. Hjorth (Berlin: SpringerVerlag), 22

Kippen, R. M., Woods, P. M., Heise, J., et al. 2003, AIPC, 662, 244 Kippen, R. M., in't Zand, J. J. M., Woods, P. M., et al. 2004, AIPC, 727, 119 Kulkarni, S. R., Frail, D. A., Wieringa, M. H., et al. 1998, Nature, 395, 663

Lamb, D. Q., Ricker, G. R., Atteia, J.-L., et al. 2003, in Proc. 3rd Rome Workshop on Gamma-Ray Bursts in the Afterglow Era [arXiv: astro-ph/0312503]

Lamb, D. Q., Donaghy, T. Q., \& Graziani, C. 2004a, New A Rev., 48, 459 Lamb, D. Q., Ricker, G. R., Atteia, J.-L., et al. 2004b, New A Rev., 48, 423 Lazzati, D., Perna, R., Flasher, J., Dwarkadas, V. V., \& Fiore, F. 2006, MNRAS, 372,1791

Levan, A., Fruchter, A., Rhoads, J., et al. 2006, ApJ, 647, 471

Liang, E., Zhang, B., Virgili, F., \& Dai, Z. G. 2007, ApJ, 662, 1111

Lloyd, N. M., Petrosian, V., \& Mallozzi, R. S. 2000, ApJ, 534, 227

Lloyd-Ronning, N. M., Fryer, C. L., \& Ramirez-Ruiz, E. 2002a, ApJ, 574, 554

Lloyd-Ronning, N. M., \& Ramirez-Ruiz, E. 2002b, ApJ, 576, 101

Maiorano, E., Masetti, N., Palazzi, E., et al. 2006, A\&A, 455, 423

Malesani, D., Tagliaferri, G., Chincarini, G., et al. 2004, ApJ, 609, L5

Martini, P., Garnavich, P., \& Stanek, K. Z. 2003, GCN Circ., 1980

Masetti, N., Palazzi, E., Pian, E., et al. 2006, GCN Circ., 4803

Matheson, T., Garnavich, P. M., Stanek, K. Z., et al. 2003, ApJ, 599, 394

Mazzali, P. A., Deng, J., Tominaga, N., et al. 2003, ApJ, 599, 95

Mirabal, N., Halpern, J. P., et al. 2006a, GCN Circ., 4792

Mirabal, N., Halpern, J. P., An, D., Thorstensen, J. R., \& Terndrup, D. M. 2006b, ApJ, 643, L99

Modjaz, M., Stanek, K. Z., Garnavich, P. M., et al. 2006, ApJ, 645, L21

Norris, J. P., Marani, G. F., \& Bonnell, J. T. 2000, ApJ, 534, 248

Pélangeon, A., \& Atteia, J.-L. 2006, GCN Circ., 5004

Pélangeon, A., \& Atteia, J.-L. 2007a, GCN Circ., 6033

Pélangeon, A., \& Atteia, J.-L. 2007b, GCN Circ., 6054

Pélangeon, A., Atteia, J.-L., Ricker, G. R., et al. 2006, in Proc. of Gamma-ray bursts in the Swift era: Sixteenth Maryland Astrophysics Conference, ed. S. S. Holt, N. Gehrels, \& J. Nousek, AIP Conf. Proc., 836, 149 Perna, R., Sari, R., \& Frail, D. 2003, ApJ, 594, 379

Pian, E., Amati, L., Antonelli, L. A., et al. 1999, A\&AS, 138, 463 Pian, E., Mazzali, P. A., Masetti, N., et al. 2006, Nature, 442, 1011 Porciani, C., \& Madau, P. 2001, ApJ, 548, 522

Preece, R. D., Briggs, M. S., Mallozzi, R. S., et al. 2000, ApJS, 126, 19 
Price, P. A., Schmidt, B. P., Kulkarni, S. R., et al. 2002, GCN Circ., 1259 Price, P. A., Fox, D. W., Djorgovski, S. G., et al. 2003, GCN Circ., 1889 Prochaska, J. X., Bloom, J. S., Chen, H.-W., et al. 2005, GCN Circ., 3204 Rau, A., Salvato, M., \& Greiner, J. 2005, A\&A, 444, 425

Reichart, D. E., Lamb, D. Q., Fenimore, E. E., et al. 2001, ApJ, 552, 57 Ricker, G. R., Atteia, J.-L., Crew, G., et al. 2001, AIPC, 662, 3

Rol, E., Vreeswijk, P., Jaunsen, A., et al. 2003, GCN Circ., 1981 Rowan-Robinson, M. 1999, Ap\&SS, 266, 291

Sakamoto, T., Lamb, D. Q., Graziani, C., et al. 2004, ApJ, 602, 875

Sakamoto, T., Lamb, D. Q., Kawai, N., et al. 2005, ApJ, 629, 311

Sakamoto, T., Barbier, L., Barthelmy, S. D., et al. 2006a, ApJ, 636, 73

Sakamoto, T., Barbier, L., Barthelmy, S. D., et al. 2006b, in Proc. of Gamma-ray bursts in the Swift era: Sixteenth Maryland Astrophysics Conference, ed. S. S. Holt, N. Gehrels, \& J. Nousek, AIP Conf. Proc., 836, 578

Savaglio, S., Fiore, F., Israel, G., et al. 2002, GCN Circ., 1633

Schaefer, B. E. 2002, Gamma-Ray Bursts: The Brightest Explosions in the Universe (Harvard)

Schaefer, B. E. 2003, ApJ, 583, L71

Schaefer, B. E. 2007, ApJ, 660, 16

Schmidt, M. 1999, ApJ, 523, L117

Schmidt, M. 2001, ApJ, 552, 36

Schmidt, M. 2004, ApJ, 616, 1072

Shin, M.-S., Berger, E., Penprase, B. E., et al. 2006, ApJ, submitted [arXiv: astro-ph/0608327]

Shirasaki, Y., Kawai, N., Yoshida, A., et al. 2003, PASJ, 55, 1033

Soderberg, A. M. 2006, in Proc. of Gamma-ray bursts in the Swift era: Sixteenth Maryland Astrophysics Conference, ed. S. S. Holt, N. Gehrels, \& J.Nousek, AIP Conf. Proc., 836, 380

Soderberg, A. M. 2007, in Proc. for Supernova 1987A: 20 Years After: Supernovae and Gamma-Ray Bursters (New York: AIPC), ed. S. Immler, K. W. Weiler, \& R. McCray [arXiv: 0706 . 3047]

Soderberg, A. M., Kulkarni, S. R., Berger, E., et al. 2004a, ApJ, 606, 994 Soderberg, A. M., Frail, D. A., \& Wieringa, M. H. 2004b, ApJ, 607, L13 Soderberg, A. M., Kulkarni, S. R., Price, P. A., et al. 2006a, ApJ, 636, 391

Soderberg, A. M., Nakar, E., Berger, E., \& Kulkarni, S. R. 2006b, ApJ, 638, 930

Soderberg, A. M., Kulkarni, S. R., Nakar, E., et al. 2006c, Nature, 442, 1014

Sollerman, J., Jaunsen, A. O., Fynbo, J. P. U., et al. 2006, A\&A, 454, 503

Stanek, K. Z., Matheson, T., Garnavich, P. M., et al. 2003, ApJ, 591, L17

Stratta, G., Basa, S., Butler, N., et al. 2007, A\&A, 461, 485

Tinney, C., Stathakis, R., Cannon, R., \& Galama, T. 1998, IAUC, 6896, 1

Vanderspek, R., \& the HETE-2 Team 2008, in prep.

Vanderspek, R., Crew, G., Doty, J., et al. 2003, GCN Circ., 1997

Vanderspek, R., Sakamoto, T., Barraud, C., et al. 2004, ApJ, 617, 1251

Villasenor, J. S., et al. 2003, in Gamma-Ray Burst and Afterglow Astronomy 2001, ed. G. R. Ricker, \& R. K. Vanderspek (Melville, NY: AIP), 33

Villasenor, J. S., Lamb, D. Q., Ricker, G. R., et al. 2005, Nature, 437, 855

Vreeswijk, P., Fruchter, A., Hjorth, J., et al. 2002, GCN Circ., 1785

Vreeswijk, P., Wijers, R., Rol, E., et al. 2003, GCN Circ., 1953

Wiersema, K., Starling, R. L. C., Rol, E., et al. 2004, GCN Circ., 2800

Woosley, S. E. 1993, ApJ, 405, 273

Woosley, S. E., \& Bloom, J. S. 2006, ARA\&A, 44, 507

Yonetoku, D., Murakami, T., Nakamura, T., et al. 2004, ApJ, 609, 935
1 Laboratoire d'Astrophysique de Toulouse-Tarbes, Université de Toulouse, CNRS, 14 Av. Edouard Belin, 31400 Toulouse, France e-mail: [alexandre.pelangeon; atteia]@ast.obs-mip.fr

2 Department of Physics and Mathematics, Aoyama Gakuin University, 5-10-1 Fuchinobe, Sagamihara, Kanagawa 229-8558, Japan

3 RIKEN (Institute of Physical and Chemical Research), 2-1 Hirosawa, Wako, Saitama 351-0198, Japan

4 Center for Space Research, Massachusetts Institute of Technology, 70 Vassar Street, Cambridge, MA, 02139, USA

5 Space Sciences Laboratory, University of California at Berkeley, 7 Gauss Way, Berkeley, CA, 94720-7450, USA

6 Department of Physics, Tokyo Institute of Technology, 2-12-1 Ookayama, Meguro-ku, Tokyo, 152-8551, Japan

7 INAF/IASF Bologna, via Gobetti 101, 40129 Bologna, Italy

8 Observatoire de Haute-Provence (CNRS/OAMP), Saint Michel l'Observatoire, France

9 Instituto Nacional de Pesquisas Espaciais, Avenida Dos Astraunotas 1758, São Jose dos Campos 12227-010, Brazil

10 Department of Astronomy and Astrophysics, University of Chicago, 5640 South Ellis Avenue, Chicago, IL 60637, USA

11 Centre d'Étude Spatiale des Rayonnements, Université de Toulouse, CNRS, 9 Av. du Colonel Roche, 31028 Toulouse, France

12 Los Alamos National Laboratory, PO Box 1663, Los Alamos, NM, 87545, USA

13 Department of Astronomy and Astrophysics, Tata Institute of Fundamental Research, Homi Bhabha Road, Mumbai, 400 005, India

14 Tsukuba Space Center, National Space Development Agency of Japan, Tsukuba, Ibaraki, 305-8505, Japan

15 NASA Goddard Space Flight Center, Greenbelt, MD, 20771, USA

16 National Astronomical Observatory, 2-21-1, Osawa, Mitaka, Tokyo, 181-8588, Japan

17 Department of Physics, Aoyama Gakuin University, Chitosedai, 616-1, Setagaya-ku, Tokyo, 157-8572, Japan

18 Faculty of Engineering, Miyazaki University, Gakuen Kibanadai Nishi, Miyazaki, 889-2192, Japan

19 Department of Astronomy and Astrophysics, Univ of California at Santa Cruz, 477 Clark Kerr Hall, Santa Cruz, CA, 95064, USA

20 Center for Research and Exploration in Space Science and Technology (CRESST), NASA Goddard Space Flight Center, Greenbelt, MD 20771, USA

21 Joint Center for Astrophysics, University of Maryland, Baltimore County, 1000 Hilltop Circle, Baltimore, MD 21250, USA 
Table 2. Observed properties and distances used in this study. The GRB names in the format $Y Y M M D D$ are given in Col. 1 . Columns 2 to 5 contain the spectral parameters measured for each burst: the slope of the power-laws at low-energy $(\alpha)$ and at high-energy when possible $(\beta)$; the peak energy $\left(E_{\text {peak }}^{\text {obs }}\right)$ in $\mathrm{keV}$ and the fluence in the energy range $2-30 \mathrm{keV}\left(S_{\mathrm{X}}\right)$, in units of $10^{-7} \mathrm{erg} \mathrm{cm}^{-2}$. The $T_{90}$ durations in the FREGATE band $B$ $(6-80 \mathrm{keV})$ are reported in Col. 6. The signal-to-noise ratio $(S N R)$ corresponding to the best combination of energy range and time resolution (see Sect. 2) are in Col. 7. The $z_{\text {sample }}$ (i.e. the spectroscopic/photometric redshifts when available or the pseudo-redshifts otherwise) are reported in Col. 8. The last column contains the references for the redshifts.

\begin{tabular}{|c|c|c|c|c|c|c|c|c|}
\hline$\overline{\text { GRB }}$ & $\alpha$ & $\beta$ & $E_{\text {peak }}^{\text {obs }}(\mathrm{keV})$ & $S_{\mathrm{X}}$ & $T_{90, \mathrm{~B}}$ & SNR & $z_{\text {sample }}$ & Ref. $z_{\text {sample }}$ \\
\hline 001226 & -1 & $-2.094_{-0.293}^{+0.194}$ & $22.32_{-7.42}^{+7.48}$ & $17.03_{-1.7}^{+1.7}$ & $90.69 \pm 2.5$ & 10.35 & $0.98_{-0.3}^{+0.3}$ & {$[0]$} \\
\hline 010213 & -1 & $-3_{-0.5}^{+0.2}$ & $3.4_{-0.4}^{+0.4}$ & $7.9_{-0.5}^{+0.3}$ & $20.12 \pm 2.16$ & 41.98 & $0.17_{-0.06}^{+0.06}$ & {$[0]$} \\
\hline 010225 & $-1.3_{-0.3}^{+0.3}$ & -- & $32_{-9}^{+27}$ & $3.5_{-0.4}^{+0.4}$ & $6.3 \pm 0.6$ & 7.94 & $1.47_{-0.55}^{+0.05}$ & {$[0]$} \\
\hline 010326 & $-1.08_{-0.22}^{+0.25}$ & -- & $51.8_{-11.3}^{+18.6}$ & $2.42_{-0.39}^{+0.39}$ & $2.15 \pm 0.32$ & 20.71 & $2.93_{-1.65}^{+1.65}$ & {$[0]$} \\
\hline 010612 & $-1.1_{-0.2}^{+0.2}$ & -- & $240_{-82}^{+290}$ & $8.8_{-1.3}^{+1.4}$ & $49.98 \pm 3.54$ & 23.52 & $5.25_{-2.2}^{+2.23}$ & {$[0]$} \\
\hline 010613 & $-1_{-0.3}^{+0.3}$ & $-2_{-0.2}^{+0.1}$ & $46_{-10}^{+18}$ & $102_{-7}^{+7}$ & $146.74 \pm 2.44$ & 45.05 & $0.64_{-0.2}^{+0.2}$ & {$[0]$} \\
\hline 010629 & $-1.1_{-0.1}^{+0.1}$ & -- & $46_{-4}^{+5}$ & $25.4_{-1.7}^{+1.7}$ & $15.8 \pm 0.28$ & 40.15 & $0.91_{-0.4}^{+0.2}$ & {$[0]$} \\
\hline 010921 & $-1.6_{-0.1}^{+0.1}$ & -- & $89_{-14}^{-22}$ & $72_{-3}^{+3.1}$ & $21.92 \pm 1.18$ & 71.52 & $0.45_{-0.005}^{+0.005}$ & {$[1 ; 2]$} \\
\hline 010928 & $-0.7_{-0.1}^{+0.1}$ & -- & $410_{-75}^{+120}$ & $13.7_{-1.4}^{+1.2}$ & $31.73 \pm 0.97$ & 39.04 & $3.64_{-0.8}^{+0.8}$ & {$[0]$} \\
\hline 011019 & -1.4 & -- & $19_{-9}^{+18}$ & $3_{-0.6}^{+0.6}$ & $22.5 \pm 2.24$ & 8.75 & $0.5_{-0.5}^{+0.5}$ & {$[0]$} \\
\hline 011130 & -- & $-2.7_{-0.3}^{+0.3}$ & $<3.9$ & $5.9_{-1}^{+1}$ & $10.64 \pm 2.86$ & 7.66 & $<0.17$ & {$[0]$} \\
\hline 011212 & -1.23 & $-2.15_{-1.33}^{+0.26}$ & $<3.74$ & $4.85_{-0.49}^{+0.49}$ & $39.62 \pm 6.55$ & 8.09 & $0.34_{-0.22}^{+0.22}$ & {$[0]$} \\
\hline 020124 & $-0.87_{-0.16}^{+0.19}$ & $-2.6_{-0.65}^{+0}$ & $82_{-17}^{+17}$ & $20_{-1.4}^{+1.4}$ & $51.17 \pm 1.55$ & 21.63 & $3.198_{-0.004}^{-0.004}$ & [3] \\
\hline 020127 & $-1_{-0.1}^{+0.1}$ & -- & $100_{-24}^{+47}$ & $6.7_{-0.5}^{+0.5}$ & $6.99 \pm 0.26$ & 27.65 & $1.9_{-0.4}^{+0.2}$ & [4] \\
\hline 020305 & $-1.06_{-0.13}^{+0.14}$ & -2.3 & $245.1_{-99.9}^{+276.7}$ & $27.49_{-4.82}^{-1.3}$ & $39.06 \pm 1.17$ & 28.54 & $1.98_{-0.7}^{+0.4}$ & {$[0]$} \\
\hline 020317 & $-0.6_{-0.5}^{-0.6}$ & -- & $28_{-7}^{+13}$ & $2.2_{-0.4}^{+4.4}$ & $7.14 \pm 1.04$ & 12.11 & $2.11_{-0.55}^{-0.55}$ & {$[0]$} \\
\hline 020331 & $-0.8_{-0.1}^{+0.1}$ & -- & $92_{-14}^{+21}$ & $16_{-1}^{+1.4}$ & $179.4 \pm 5.99$ & 28.15 & $2.21_{-0.7}^{+0.03}$ & {$[0]$} \\
\hline 020625 & -1.1 & -- & $8.5_{-2.9}^{+5.4}$ & $2.4_{-0.5}^{+0.6}$ & $13.98 \pm 3.82$ & 8.18 & $0.73_{-0.13}^{+0.13}$ & {$[0]$} \\
\hline 020801 & $-0.3_{-0.3}^{+0.4}$ & $-2_{-0.3}^{+0.2}$ & $53_{-11}^{+14}$ & $26_{-3}^{+3}$ & $460.33 \pm 3.63$ & 15.54 & $1.21_{-1}^{+1}$ & {$[0]$} \\
\hline 020812 & $-1.1_{-0.3}^{+0.3}$ & -- & $88_{-30}^{+110}$ & $8_{-1}^{+1}$ & $18.28 \pm 1.55$ & 14.63 & $3.48_{-1.8}^{+1.8}$ & {$[0]$} \\
\hline 020813 & $-1.36_{-0.05}^{+0.05}$ & -2.3 & $253.4_{-35.6}^{+52.4}$ & $147.91_{-7}^{+7.32}$ & $87.34 \pm 0.6$ & 92.98 & $1.255_{-0.005}^{+0.005}$ & [5] \\
\hline 020819 & $-0.9_{-0.1}^{+0.2}$ & $-2_{-0.5}^{+0.2}$ & $\begin{array}{l}50_{-12}^{+18} \\
x^{+3.0}\end{array}$ & $25.2_{-1.1}^{+1.1}$ & $28.8 \pm 7.19$ & 50.61 & $0.41_{-0.01}^{+0.01}$ & [6] \\
\hline 021004 & $-1_{-0.2}^{+0.2}$ & -- & $80_{-23}^{+53}$ & $7.7_{-0.7}^{+0.7}$ & $48.94 \pm 2.5$ & 13.85 & $2.31_{-0.01}^{+0.01}$ & {$[7 ; 8 ; 9]$} \\
\hline 021016 & $-1.2_{-0.12}^{+0.11}$ & -2.3 & $226.2_{-86.4}^{+268.7}$ & $34.17_{-3.59}^{+2.44}$ & $80.63 \pm 0.95$ & 28.55 & $2.8_{-1.6}^{+1.61}$ & {$[0]$} \\
\hline 021021 & -1.3 & -- & $15_{-8}^{+14}$ & $2.5_{-0.6}^{+0.6}$ & $18.9 \pm 5.13$ & 6.28 & $1_{-0.5}^{+0.5}$ & {$[0]$} \\
\hline 021104 & $-1.1_{-0.5}^{+0.5}$ & -- & $28_{-8}^{+17}$ & $10_{-2}^{+2}$ & $21.18 \pm 4.08$ & 10.52 & $1.1_{-0.3}^{+0.3}$ & {$[0]$} \\
\hline 021112 & $-0.9_{-0.3}^{+0.4}$ & -- & $57_{-21}^{+39}$ & $1.3_{-0.3}^{+0.3}$ & $3.24 \pm 1.28$ & 9.86 & $4.15_{-1.9}^{+1.9}$ & {$[0]$} \\
\hline 021211 & $-0.805_{-0.105}^{+0.112}$ & $-2.37_{-0.31}^{+0.18}$ & $46.8_{-5.1}^{+5.8}$ & $13.6_{-0.5}^{+0.5}$ & $4.23 \pm 0.27$ & 76.24 & $1.006_{-0.001}^{+0.001}$ & {$[10 ; 11]$} \\
\hline 030115 & $-1.3_{-0.1}^{+0.1}$ & -- & $83_{-22}^{+53}$ & $7.9_{-0.6}^{+0.6}$ & $20.33 \pm 3.54$ & 27.29 & $2.2_{-0.1}^{+0.1}$ & {$[12]$} \\
\hline 030226 & $-0.9_{-0.2}^{+0.2}$ & -- & $97_{-17}^{+27}$ & $13_{-1}^{+1}$ & $76.23 \pm 3.96$ & 16.3 & $1.98_{-0.02}^{+0.02}$ & {$[13 ; 14 ; 15 ; 16 ; 17]$} \\
\hline 030324 & $-1.5_{-0.1}^{+0.1}$ & -- & $150_{-65}^{+630}$ & $5.5_{-0.4}^{+0.4}$ & $10.98 \pm 2.84$ & 21.27 & $>2.3$ & {$[0]$} \\
\hline 030328 & $-1.14_{-0.03}^{+0.03}$ & $-2.1_{-0.4}^{+0.2}$ & $130_{-13}^{+14}$ & $82_{-1}^{+1}$ & $138.27 \pm 3.05$ & 55.68 & $1.5216_{-0.0006}^{+0.0006}$ & {$[18 ; 19 ; 20 ; 21]$} \\
\hline 030329 & $-1.32_{-0.02}^{+0.02}$ & $-2.44_{-0.08}^{+0.08}$ & $70.2_{-2.3}^{+2.3}$ & $576_{-5}^{+5}$ & $25.91 \pm 0.39$ & 581.25 & $0.1685_{-0.0001}^{+0.0001}$ & {$[22 ; 23 ; 24]$} \\
\hline 030416 & -- & $-2.3_{-0.2}^{+0.10}$ & $2.6_{-1.8}^{-0.5}$ & $9_{-0.9}^{+0.9}$ & $14.29 \pm 2.29$ & 18.02 & $0.11_{-0.04}^{+0.04}$ & [0] \\
\hline 030418 & $-1.5_{-0.1}^{+0.1}$ & -- & $46_{-14}^{+32}$ & $17.1_{-1.1}^{+1.1}$ & $139.23 \pm 7.76$ & 9.26 & $3.07_{-1.7}^{+1.7}$ & [0] \\
\hline 030429 & $-1.1_{-0.2}^{+0.3}$ & -- & $35_{-8}^{+12}$ & $4.7_{-0.5}^{+0.5}$ & $12.95 \pm 2.69$ & 13.25 & $2.658_{-0.004}^{+0.004}$ & [25] \\
\hline 030519 & $-0.8_{-0.1}^{+0.1}$ & $-1.7_{-0.1}^{+0.1}$ & $138_{-15}^{+18}$ & $87.1_{-2.4}^{+2.4}$ & $12.85 \pm 0.56$ & 193.85 & $0.86_{-0.1}^{+0.1}$ & {$[0]$} \\
\hline 030528 & $-1.3_{-0.1}^{+0.1}$ & $-2.7_{-1}^{+0.3}$ & $32_{-5}^{+5}$ & $62_{-3}^{+3}$ & $62.8 \pm 4.49$ & 28.11 & $0.782_{-0.001}^{+0.001}$ & [26] \\
\hline 030723 & -- & $-1.9_{-0.2}^{+0.2}$ & $<8.9$ & $2.8_{-0.5}^{+0.5}$ & $9.63 \pm 1.5$ & 9.74 & $0.54_{-0.15}^{+0.15}$ & {$[0]$} \\
\hline 030725 & $-1.51_{-0.04}^{+0.04}$ & --0.2 & $102_{-14}^{+19}$ & $94_{-2}^{+2}$ & $174.31 \pm 17.07$ & 88.56 & $0.89_{-0.2}^{+0.13}$ & {$[0]$} \\
\hline 030821 & $-0.9_{-0.1}^{+0.1}$ & -- & $84_{-11}^{+15}$ & $10_{-0.6}^{+0.6}$ & $19.42 \pm 0.44$ & 21.43 & $1.77_{-0.5}^{+0.5}$ & {$[0]$} \\
\hline 030823 & $-1.3_{-0.2}^{+0.1}$ & -- & $27_{-5}^{+8}$ & $23.1_{-1.6}^{+1.6}$ & $50.39 \pm 3.11$ & 15.49 & $0.83_{-0.3}^{+0.3}$ & {$[0]$} \\
\hline 030824 & -- & $-2.1_{-0.1}^{+0.1}$ & $6.1_{-4.2}^{+1.9}$ & $8.9_{-1.1}^{+1.0}$ & $10.13 \pm 1.04$ & 12.84 & $0.26_{-0.1}^{+0.1}$ & {$[0]$} \\
\hline 030913 & $-0.8_{-0.2}^{+0.3}$ & --0 & $120_{-37}^{+110}$ & $1.8_{-0.2}^{-0.1}$ & $6.58 \pm 2.3$ & 14.23 & $6.04_{-2.7}^{+2.7}$ & {$[0]$} \\
\hline 031026 & $-1.13_{-0.21}^{+0.26}$ & -2.3 & $870.3_{-679}^{+129.7}$ & $3.56_{-3.46}^{+1.26}$ & $65.58 \pm 4.55$ & 8.46 & $\begin{array}{l}6.67_{-2.9}^{+2.9} \\
\end{array}$ & {$[0]$} \\
\hline 031109A & $-1.17_{-0.04}^{+0.03}$ & -2.3 & $185.2_{-23.7}^{+29.8}$ & $98.3_{-2.9}^{+3.40}$ & $57.32 \pm 0.46$ & 78.68 & $0.94_{-0.2}^{+0.2}$ & {$[0]$} \\
\hline 031109B & $-1.27_{-0.24}^{+0.28}$ & -- & $37.7_{-12.29}^{+28.4}$ & $4.9_{-0.6}^{+0.5}$ & $531.25 \pm 72.25$ & 8.7 & $2.05_{-0.9}^{+0.9}$ & [0] \\
\hline 031111A & $-0.82_{-0.05}^{-0.05}$ & -- & $404.4_{-51.4}^{+67.8}$ & $14.9_{-0.7}^{+0.0 .6}$ & $7.94 \pm 0.53$ & 151.81 & $2.14_{-0.4}^{+0.4}$ & {$[0]$} \\
\hline 031111B & --0.03 & $-2.2_{-0.38}^{+0.2}$ & $6.01_{-5.01}^{+3.8}$ & $9.85_{-0.99}^{+0.99}$ & $27.34 \pm 2.16$ & 5.81 & $0.11_{-0.17}^{+0.17}$ & {$[0]$} \\
\hline 031203 & $-1.18_{-0.09}^{+0.1}$ & -2.3 & $148.2_{-23.91}^{+32.45}$ & $22.68_{-1.68}^{+1.44}$ & $10.38 \pm 0.29$ & 92.35 & $2.17_{-0.7}^{+0.7}$ & {$[0]$} \\
\hline
\end{tabular}


Table 2. continued.

\begin{tabular}{|c|c|c|c|c|c|c|c|c|}
\hline GRB & $\alpha$ & $\beta$ & $E_{\text {peak }}^{\text {obs }}(\mathrm{keV})$ & $S_{\mathrm{X}}$ & $T_{90, \mathrm{~B}}$ & SNR & $z_{\text {sample }}$ & Ref. $z_{\text {sample }}$ \\
\hline 031220 & $-1.51_{-0.2}^{+0.23}$ & $-3.25_{-6.75}^{+1.3}$ & $46.9_{-16.4}^{+62.1}$ & $5.5_{-0.5}^{+0.6}$ & $9.69 \pm 0.93$ & 13.9 & $1.53_{-1.2}^{+1.2}$ & {$[0]$} \\
\hline 040319 & $-0.89_{-0.2}^{+0.24}$ & -- & $56.6_{-9.7}^{+14.1}$ & $6_{-0.5}^{+0.6}$ & $6.1 \pm 0.85$ & 23.12 & $1.79_{-1.2}^{+1.2}$ & [0] \\
\hline 040423 & $-1.02_{-0.27}^{+0.31}$ & -- & $30.7_{-4}^{+4.8}$ & $22.7_{-2.2}^{+2.6}$ & $45.87 \pm 1.13$ & 17.47 & $1.26_{-0.7}^{+0.7}$ & [0] \\
\hline 040425 & $-0.86_{-0.08}^{+0.09}$ & -- & $299.9_{-48.7}^{+72.9}$ & $17.2_{-1.4}^{+1.2}$ & $138.81 \pm 1.65$ & 11.16 & $2.23_{-0.5}^{+0.5}$ & [0] \\
\hline 040511 & $-0.92_{-0.14}^{+0.15}$ & -- & $93.9_{-11.3}^{+15.7}$ & $31.7_{-2.8}^{+0.3}$ & $45.8 \pm 0.53$ & 36.61 & $1.83_{-0.5}^{+0.5}$ & [0] \\
\hline 040701 & -- & $-2.3_{-0.31}^{+0.25}$ & $<3.44$ & $5.44_{-0.55}^{+0.55}$ & $11.67 \pm 5.72$ & 12.76 & $0.2146_{-0.0005}^{+0.0005}$ & [27] \\
\hline 040709 & $-1.25_{-0.07}^{+0.08}$ & -- & $70.9_{-6.7}^{+8.3}$ & $80.6_{-4}^{+4.2}$ & $82.48 \pm 0.24$ & 58.3 & $1_{-0.3}^{+0.3}$ & [0] \\
\hline 040802 & $-0.85_{-0.2}^{+0.23}$ & -- & $92.2_{-13}^{+18.8}$ & $4.79_{-0.68}^{+0.68}$ & $3.05 \pm 0.18$ & 39.39 & $1.81_{-0.65}^{+0.65}$ & [0] \\
\hline 040825A & $-1.51_{-0.22}^{+0.22}$ & -- & $60_{-30}^{+30}$ & $9.6_{-1.5}^{+1.5}$ & $39.2 \pm 2.01$ & 10.47 & $1.06_{-0.5}^{+0.5}$ & [0] \\
\hline 040825B & $-1.48_{-0.17}^{+0.21}$ & -- & $25.1_{-8.2}^{+15.6}$ & $12_{-0.9}^{+0.9}$ & $15.8 \pm 5.45$ & 10.3 & $2.2_{-0.8}^{+0.8}$ & [0] \\
\hline 040912A & $-1.17_{-0.62}^{+1.54}$ & $-2.49_{-0.4}^{+0.24}$ & $14.16_{-4.32}^{+3.32}$ & $20.36_{-2.04}^{+2.04}$ & $9.21 \pm 0.27$ & 39.44 & $0.33_{-0.3}^{+0.3}$ & [0] \\
\hline 040912B & $-1.25_{-0.58}^{+0.82}$ & -- & $17_{-13}^{+13}$ & $9.5_{-0.95}^{+0.95}$ & $122.41 \pm 7.18$ & 8.19 & $1.563_{-0.001}^{+0.001}$ & [28] \\
\hline 040916 & -1 & -- & $<3.5$ & $7.74_{-0.91}^{+0.91}$ & $349 \pm 10.23$ & 6.66 & $<0.7$ & [0] \\
\hline 040924 & $-1.03_{-0.09}^{+0.09}$ & -- & $41.1_{-2.3}^{+2.3}$ & $23.4_{-0.24}^{+0.24}$ & $3.37 \pm 0.08$ & 85.97 & $0.859_{-0.005}^{+0.005}$ & [29] \\
\hline 041004 & $-1.3_{-0.17}^{+1.17}$ & -- & $53.7_{-7.5}^{+11.7}$ & $133.8_{-18.4}^{+19.7}$ & $50.13 \pm 2.49$ & 35.64 & $0.58_{-0.2}^{+0.2}$ & [0] \\
\hline 041006 & $-1.3_{-0.05}^{+0.05}$ & -- & $47.7_{-2.7}^{+3}$ & $38.9_{-0.9}^{+0.6}$ & $22.08 \pm 0.33$ & 120.37 & $0.716_{-0.005}^{+0.005}$ & [30] \\
\hline 041016 & $-1.13_{-0.21}^{+0.36}$ & -2.3 & $165.3_{-75.2}^{+731.8}$ & $8.21_{-1.93}^{+1.53}$ & $21.96 \pm 1.7$ & 13.59 & $3.49_{-1.7}^{+1.7}$ & [0] \\
\hline 041127 & $-1.01_{-0.16}^{+0.27}$ & $-2.27_{-0.47}^{+0.24}$ & $35_{-10.2}^{+10}$ & $26.9_{-1.4}^{+0.07}$ & $49.14 \pm 2.26$ & 25.19 & $0.96_{-0.5}^{+0.5}$ & [0] \\
\hline 041211 & $-0.69_{-0.2}^{+0.48}$ & $-1.48_{-0.54}^{+0.16}$ & $132_{-76.3}^{+116}$ & $10.6_{-0.8}^{+0.4}$ & $113.84 \pm 4.2$ & 44.38 & $3.29_{-0.9}^{+0.9}$ & [0] \\
\hline 050123 & $-0.71_{-0.2}^{+0.22}$ & -- & $40.2_{-5}^{+6}$ & $6.7_{-3.7}^{+0.5}$ & $14.72 \pm 1.17$ & 25.06 & $1.53_{-0.85}^{+0.85}$ & [0] \\
\hline 050209 & $-1.52_{-0.15}^{+0.3}$ & -2.3 & $445_{-350.5}^{+555}$ & $5.5_{-5.4}^{+0.82}$ & $18.38 \pm 0.59$ & 15.37 & $2.93_{-1.6}^{+1.6}$ & [0] \\
\hline 050408 & $-1.76_{-0.06}^{+0.09}$ & $-2.2_{-0.28}^{+0.16}$ & $25.9_{-7.3}^{+10.5}$ & $32.4_{-1.1}^{+0.3}$ & $28.39 \pm 0.56$ & 50.85 & $1.2357_{-0.0002}^{+0.0002}$ & {$[31 ; 32]$} \\
\hline 050509 & -- & -- & $<19$ & $6_{-0.6}^{+0.6}$ & $21 \pm 5$ & 7.06 & $0.68_{-03}^{+0.302}$ & {$[0]$} \\
\hline 050729 & $-0.61_{-0.32}^{+0.43}$ & -2.3 & $78.42_{-16.03}^{+23.22}$ & $3.97_{-0.65}^{+0.69}$ & $5.23 \pm 0.51$ & 24.35 & $2.5_{-0.75}^{+0.75}$ & [0] \\
\hline 050807 & $-1.53_{-0.22}^{+0.42}$ & -2.3 & $69.47_{-31.12}^{+10.9}$ & $14.15_{-3.36}^{+1.63}$ & $10.32 \pm 1$ & 19.17 & $0.79_{-0.75}^{+0.75}$ & {$[0]$} \\
\hline 050922 & $-0.83_{-0.26}^{+0.23}$ & -- & $130.5_{-26.8}^{+50.9}$ & $5.4_{-0.54}^{+0.54}$ & $6.13 \pm 1.4$ & 38.25 & $2.198_{-0.001}^{+0.001}$ & [33] \\
\hline 051021 & $-1.04_{-0.1}^{+0.11}$ & -- & $96_{-16.6}^{+26}$ & $19.2_{-0.7}^{+0.54}$ & $36.79 \pm 1.13$ & 35.54 & $1.37_{-0.5}^{+0.5}$ & {$[0]$} \\
\hline 051022 & $-1.01_{-0.03}^{+0.02}$ & $-1.95_{-0.14}^{+0.25}$ & $213_{-18}^{+18}$ & $214_{-2}^{+2}$ & $178 \pm 8$ & 226.38 & $0.8_{-0.01}^{+0.01}$ & [34] \\
\hline 051028 & $-0.94_{-0.16}^{+0.18}$ & -- & $249_{-70}^{+167}$ & $7.99_{-0.67}^{+0.59}$ & $14.66 \pm 1$ & 25.35 & $3.66_{-1.8}^{+1.8}$ & {$[0]$} \\
\hline 051211 & $-0.07_{-0.41}^{+0.5}$ & -- & $121_{-20.3}^{+33}$ & $0.765_{-0.228}^{+0.228}$ & $4.9 \pm 0.61$ & 18.83 & $4.83_{-1.9}^{+1.9}$ & [0] \\
\hline 060115 & $-0.74_{-0.1}^{+0.12}$ & $-1.81_{-0.21}^{+0.12}$ & $94.6_{-17.6}^{+21.4}$ & $22.4_{-0.5}^{+0.4}$ & $20.68 \pm 0.45$ & 87.09 & $1.52_{-0.25}^{+0.25}$ & [0] \\
\hline 060121 & $-0.79_{-0.11}^{+0.12}$ & -- & $114_{-10.9}^{+14.2}$ & $8.98_{-0.67}^{+0.67}$ & $2.61 \pm 0.1$ & 80.37 & $1.92_{-0.35}^{+0.35}$ & {$[0]$} \\
\hline
\end{tabular}

[0] = our work; [1] = Djorgovski (2001); [2] = Price et al. (2002); [3] = Hjorth et al. (2003b); [4] = Berger et al. (2007); [5] = Barth et al. (2003); [6] = Jakobsson et al. (2005a); [7] = Savaglio et al. (2002); [8] = Castro-Tirado et al. (2002); [9] = Lazzati et al. (2006); [10] = Vreeswijk et al. (2002); [11] = Fox et al. (2003); [12] = Levan et al. (2006); [13] = Ando et al. (2003a); [14] = Ando et al. (2003b); [15] = Greiner et al. (2003a); [16] = Price et al. (2003); [17] = Shin et al. (2006); [18] = Martini et al. (2003); [19] = Rol et al. (2003); [20] = Fugazza et al. (2003); [21] = Maiorano et al. (2006); [22] = Greiner et al. (2003b); [23] = Caldwell et al. (2003); [24] = Bloom et al. (2003); [25] = Jakobsson et al. (2004); [26] = Rau et al. (2005); [27] = Kelson et al. (2004); [28] = Stratta et al. (2007); [29] = Wiersema et al. (2004); [30] = Fugazza et al. (2004); [31] = Berger et al. (2005); [32] = Prochaska et al. (2005); [33] = Jakobsson et al. (2005b); [34] = Gal-Yam et al. (2005). 
Table 3. Intrinsic properties of our complete sample of HETE-2 localized GRBs. For each burst, the values reported in Cols. 2-4 are base 10 logarithms which enable us to obtain the individual lognormal distributions of the $E_{\text {peak }}, T_{90, \mathrm{~B}}$ and $E_{\text {iso }}$. The $z_{\max }$ used to derive the corresponding $V_{\max }$ are shown in Col. 5. The resulting $V / V_{\max }$ are reported in the last column.

\begin{tabular}{|c|c|c|c|c|c|}
\hline GRB & $\log E_{\text {peak }}^{\text {intr }}$ & $\log T_{90, \mathrm{~B}}^{\text {intr }}$ & $\log E_{\mathrm{iso}, 52}$ & $z_{\max }$ & $V / V_{\max }$ \\
\hline $001226 \ldots \ldots$ & $1.648 \pm 0.091$ & $1.66 \pm 0.037$ & $0.516 \pm 0.112$ & $1.12 \pm 0.19$ & $0.779 \pm 0.012$ \\
\hline $010213 \ldots \ldots$ & $0.593 \pm 0.03$ & $1.239 \pm 0.028$ & $-2.172 \pm 0.023$ & $0.39 \pm 0.08$ & $0.091 \pm 0.009$ \\
\hline $010225 \ldots \ldots$ & $2.003 \pm 0.122$ & $0.403 \pm 0.059$ & $-0.173 \pm 0.08$ & $1.5 \pm 0.3$ & 1 \\
\hline $010326 \ldots \ldots$ & $2.341 \pm 0.111$ & $-0.264 \pm 0.106$ & $0.518 \pm 0.115$ & $4.85 \pm 1.47$ & $0.552 \pm 0.049$ \\
\hline $010612 \ldots \ldots$ & $3.306 \pm 0.168$ & $0.916 \pm 0.095$ & $1.457 \pm 0.11$ & $9.22 \pm 2.2$ & $0.61 \pm 0.052$ \\
\hline $010613 \ldots \ldots$ & $1.891 \pm 0.087$ & $1.952 \pm 0.034$ & $0.711 \pm 0.12$ & $1.42 \pm 0.28$ & $0.173 \pm 0.016$ \\
\hline $010629 \ldots \ldots$ & $1.938 \pm 0.06$ & $0.925 \pm 0.053$ & $0.357 \pm 0.054$ & $1.86 \pm 0.49$ & $0.228 \pm 0.029$ \\
\hline $010921 \ldots \ldots$. & $2.128 \pm 0.053$ & $1.177 \pm 0.014$ & $-0.076 \pm 0.028$ & $1.18 \pm 0.01$ & $0.104 \pm 0.001$ \\
\hline $010928 \ldots \ldots$ & $3.293 \pm 0.073$ & $0.841 \pm 0.048$ & $1.814 \pm 0.12$ & $9.23 \pm 1.43$ & $0.406 \pm 0.027$ \\
\hline $011019 \ldots \ldots$ & $1.525 \pm 0.178$ & $1.168 \pm 0.089$ & $-1.324 \pm 0.123$ & $0.65 \pm 0.34$ & $0.63 \pm 0.038$ \\
\hline $011130 \ldots \ldots$ & $0.43 \pm 0.162$ & $0.958 \pm 0.068$ & $-2.26 \pm 0.06$ & $0.19 \pm 0.05$ & $0.705 \pm 0.038$ \\
\hline $011212 \ldots \ldots$ & $0.471 \pm 0.177$ & $1.476 \pm 0.063$ & $-1.703 \pm 0.061$ & $0.38 \pm 0.16$ & $0.679 \pm 0.028$ \\
\hline $020124 \ldots \ldots$ & $2.531 \pm 0.053$ & $1.086 \pm 0.008$ & $1.999 \pm 0.083$ & $5.58 \pm 0.06$ & $0.532 \pm 0.005$ \\
\hline $020127 \ldots \ldots$ & $2.481 \pm 0.082$ & $0.399 \pm 0.032$ & $0.553 \pm 0.059$ & $3.45 \pm 0.38$ & $0.376 \pm 0.016$ \\
\hline $020305 \ldots \ldots$ & $2.995 \pm 0.17$ & $1.118 \pm 0.058$ & $1.22 \pm 0.107$ & $4.11 \pm 0.91$ & $0.367 \pm 0.03$ \\
\hline $020317 \ldots \ldots$ & $1.98 \pm 0.093$ & $0.36 \pm 0.06$ & $0.23 \pm 0.185$ & $2.59 \pm 0.41$ & $0.727 \pm 0.016$ \\
\hline $020331 \ldots \ldots$ & $2.48 \pm 0.077$ & $1.755 \pm 0.057$ & $1.189 \pm 0.073$ & $3.83 \pm 0.74$ & $0.455 \pm 0.032$ \\
\hline $020625 \ldots \ldots$ & $1.141 \pm 0.135$ & $0.991 \pm 0.096$ & $-1.498 \pm 0.126$ & $0.53 \pm 0.29$ & $0.671 \pm 0.035$ \\
\hline $020801 \ldots \ldots$. & $2.054 \pm 0.129$ & $2.339 \pm 0.117$ & $1.241 \pm 0.222$ & $1.68 \pm 0.85$ & $0.507 \pm 0.049$ \\
\hline $020812 \ldots \ldots$ & $2.752 \pm 0.182$ & $0.606 \pm 0.103$ & $1.063 \pm 0.121$ & $4.78 \pm 1.39$ & $0.716 \pm 0.035$ \\
\hline $020813 \ldots \ldots$ & $2.773 \pm 0.042$ & $1.588 \pm 0.002$ & $1.982 \pm 0.016$ & $3.8 \pm 0.03$ & $0.169 \pm 0.002$ \\
\hline $020819 \ldots \ldots$ & $1.858 \pm 0.079$ & $1.304 \pm 0.067$ & $-0.251 \pm 0.076$ & $0.96 \pm 0.02$ & $0.124 \pm 0.002$ \\
\hline $021004 \ldots \ldots$ & $2.492 \pm 0.104$ & $1.17 \pm 0.013$ & $0.754 \pm 0.087$ & $3.1 \pm 0.02$ & $0.655 \pm 0.006$ \\
\hline $021016 \ldots \ldots$ & $3.037 \pm 0.17$ & $1.358 \pm 0.115$ & $1.517 \pm 0.096$ & $5.01 \pm 1.83$ & $0.451 \pm 0.064$ \\
\hline $021021 \ldots \ldots$. & $1.56 \pm 0.16$ & $0.961 \pm 0.102$ & $-0.751 \pm 0.143$ & $1.08 \pm 0.3$ & $0.927 \pm 0.009$ \\
\hline $021104 \ldots \ldots$ & $1.84 \pm 0.109$ & $1.002 \pm 0.06$ & $0.097 \pm 0.116$ & $1.25 \pm 0.21$ & $0.77 \pm 0.012$ \\
\hline $021112 \ldots \ldots$ & $2.517 \pm 0.148$ & $-0.216 \pm 0.137$ & $0.482 \pm 0.168$ & $4.74 \pm 1.22$ & $0.875 \pm 0.015$ \\
\hline $021211 \ldots \ldots$ & $1.977 \pm 0.03$ & $0.321 \pm 0.015$ & $0.348 \pm 0.043$ & $2.93 \pm 0.02$ & $0.149 \pm 0.002$ \\
\hline $030115 \ldots \ldots$ & $2.484 \pm 0.094$ & $0.802 \pm 0.043$ & $0.592 \pm 0.03$ & $4.04 \pm 0.11$ & $0.436 \pm 0.006$ \\
\hline $030226 \ldots \ldots$ & $2.481 \pm 0.053$ & $1.409 \pm 0.014$ & $0.923 \pm 0.065$ & $2.9 \pm 0.03$ & $0.556 \pm 0.004$ \\
\hline $030324 \ldots \ldots$ & $3.237 \pm 0.255$ & $0.374 \pm 0.097$ & $0.847 \pm 0.035$ & $6.05 \pm 1.26$ & $0.586 \pm 0.032$ \\
\hline $030328 \ldots \ldots$ & $2.515 \pm 0.029$ & $1.739 \pm 0.005$ & $1.381 \pm 0.013$ & $4.08 \pm 0.02$ & $0.226 \pm 0.001$ \\
\hline $030329 \ldots \ldots$ & $1.915 \pm 0.008$ & $1.345 \pm 0.004$ & $0.03 \pm 0.008$ & $1.16 \pm 0.00$ & $0.007 \pm 0.000$ \\
\hline $030416 \ldots \ldots$ & $0.295 \pm 0.173$ & $1.1 \pm 0.042$ & $-2.501 \pm 0.139$ & $0.21 \pm 0.03$ & $0.228 \pm 0.01$ \\
\hline $030418 \ldots \ldots$ & $2.309 \pm 0.147$ & $1.558 \pm 0.113$ & $1.175 \pm 0.055$ & $3.19 \pm 1.07$ & $0.906 \pm 0.015$ \\
\hline $030429 \ldots \ldots$ & $2.122 \pm 0.069$ & $0.554 \pm 0.054$ & $0.54 \pm 0.049$ & $3.39 \pm 0.02$ & $0.718 \pm 0.004$ \\
\hline $030519 \ldots \ldots$ & $2.409 \pm 0.036$ & $0.841 \pm 0.018$ & $1.21 \pm 0.067$ & $3.74 \pm 0.29$ & $0.075 \pm 0.005$ \\
\hline $030528 \ldots \ldots$ & $1.753 \pm 0.043$ & $1.549 \pm 0.02$ & $0.483 \pm 0.035$ & $1.34 \pm 0.01$ & $0.307 \pm 0.003$ \\
\hline $030723 \ldots \ldots$ & $0.793 \pm 0.279$ & $0.795 \pm 0.045$ & $-1.258 \pm 0.105$ & $0.6 \pm 0.09$ & $0.802 \pm 0.013$ \\
\hline $030725 \ldots \ldots$ & $2.282 \pm 0.045$ & $1.965 \pm 0.036$ & $0.752 \pm 0.016$ & $2.6 \pm 0.33$ & $0.132 \pm 0.012$ \\
\hline $030821 \ldots \ldots$ & $2.372 \pm 0.063$ & $0.845 \pm 0.048$ & $0.719 \pm 0.058$ & $3 \pm 0.53$ & $0.447 \pm 0.024$ \\
\hline $030823 \ldots \ldots$ & $1.717 \pm 0.071$ & $1.439 \pm 0.043$ & $0.101 \pm 0.054$ & $1.14 \pm 0.23$ & $0.53 \pm 0.022$ \\
\hline $030824 \ldots \ldots$ & $0.74 \pm 0.17$ & $0.915 \pm 0.041$ & $-1.659 \pm 0.089$ & $0.29 \pm 0.1$ & $0.551 \pm 0.03$ \\
\hline $030913 \ldots \ldots$ & $2.996 \pm 0.147$ & $-0.012 \pm 0.137$ & $1.023 \pm 0.189$ & $8.19 \pm 2.21$ & $0.772 \pm 0.032$ \\
\hline $031026 \ldots \ldots$ & $3.595 \pm 0.234$ & $0.947 \pm 0.093$ & $2.178 \pm 0.136$ & $6.89 \pm 1.69$ & $0.969 \pm 0.004$ \\
\hline 031109A....... & $2.564 \pm 0.044$ & $1.47 \pm 0.024$ & $1.02 \pm 0.025$ & $2.93 \pm 0.38$ & $0.129 \pm 0.011$ \\
\hline 031109B...... & $2.231 \pm 0.174$ & $2.145 \pm 0.129$ & $0.664 \pm 0.099$ & $3.09 \pm 1.18$ & $0.94 \pm 0.01$ \\
\hline 031111A...... & $3.116 \pm 0.045$ & $0.402 \pm 0.037$ & $1.288 \pm 0.053$ & $10.51 \pm 1.17$ & $0.182 \pm 0.018$ \\
\hline 031111B...... & $0.752 \pm 0.272$ & $1.359 \pm 0.027$ & $-1.808 \pm 0.14$ & $0.19 \pm 0.05$ & 1 \\
\hline $031203 \ldots \ldots$ & $2.683 \pm 0.074$ & $0.517 \pm 0.057$ & $1.661 \pm 0.057$ & $6.63 \pm 1.31$ & $0.26 \pm 0.034$ \\
\hline $031220 \ldots \ldots$ & $2.204 \pm 0.19$ & $0.585 \pm 0.117$ & $0.013 \pm 0.062$ & $2.08 \pm 0.82$ & $0.64 \pm 0.048$ \\
\hline $040319 \ldots \ldots$ & $2.183 \pm 0.129$ & $0.37 \pm 0.124$ & $0.5 \pm 0.13$ & $2.9 \pm 1.31$ & $0.421 \pm 0.062$ \\
\hline $040423 \ldots \ldots$ & $1.845 \pm 0.085$ & $1.314 \pm 0.078$ & $0.607 \pm 0.102$ & $1.8 \pm 0.58$ & $0.507 \pm 0.041$ \\
\hline $040425 \ldots \ldots$ & $3.001 \pm 0.064$ & $1.634 \pm 0.038$ & $1.307 \pm 0.07$ & $2.73 \pm 0.35$ & $0.739 \pm 0.009$ \\
\hline $040511 \ldots \ldots$ & $2.421 \pm 0.062$ & $1.22 \pm 0.05$ & $1.255 \pm 0.076$ & $3.46 \pm 0.63$ & $0.366 \pm 0.03$ \\
\hline $040701 \ldots \ldots$ & $0.405 \pm 0.146$ & $0.955 \pm 0.122$ & $-2.096 \pm 0.074$ & $0.3 \pm 0.01$ & $0.384 \pm 0.013$ \\
\hline $040709 \ldots \ldots$ & $2.15 \pm 0.051$ & $1.618 \pm 0.041$ & $0.898 \pm 0.035$ & $2.49 \pm 0.5$ & $0.182 \pm 0.021$ \\
\hline $040802 \ldots \ldots$ & $2.43 \pm 0.077$ & $0.033 \pm 0.062$ & $0.955 \pm 0.115$ & $3.74 \pm 0.79$ & $0.358 \pm 0.036$ \\
\hline 040825A....... & $2.056 \pm 0.133$ & $1.287 \pm 0.068$ & $-0.088 \pm 0.069$ & $1.17 \pm 0.35$ & $0.772 \pm 0.02$ \\
\hline 040825B...... & $1.942 \pm 0.123$ & $0.701 \pm 0.098$ & $0.698 \pm 0.039$ & $2.49 \pm 0.52$ & $0.838 \pm 0.014$ \\
\hline 040912A....... & $1.247 \pm 0.095$ & $0.851 \pm 0.064$ & $-0.782 \pm 0.24$ & $0.61 \pm 0.36$ & $0.162 \pm 0.043$ \\
\hline 040912B...... & $1.62 \pm 0.194$ & $1.68 \pm 0.015$ & $0.327 \pm 0.131$ & $1.82 \pm 0.01$ & $0.754 \pm 0.007$ \\
\hline $040916 \ldots \ldots$ & $0.465 \pm 0.163$ & $2.401 \pm 0.052$ & $-1.329 \pm 0.052$ & $0.42 \pm 0.18$ & $0.856 \pm 0.028$ \\
\hline
\end{tabular}


A. Pélangeon et al.: Intrinsic properties of a complete sample of HETE-2 GRBs, Online Material p 4

Table 3. continued.

\begin{tabular}{lccccc}
\hline \hline GRB & $\log E_{\text {peak }}^{\text {intr }}$ & $\log T_{90, \mathrm{~B}}^{\text {intr }}$ & $\log E_{\text {iso }, 52}$ & $z_{\max }$ & $V / V_{\max }$ \\
\hline $040924 \ldots \ldots$ & $1.88 \pm 0.014$ & $0.259 \pm 0.006$ & $0.292 \pm 0.029$ & $2.5 \pm 0.02$ & $0.129 \pm 0.001$ \\
$041004 \ldots \ldots$. & $1.938 \pm 0.059$ & $1.504 \pm 0.037$ & $0.632 \pm 0.235$ & $1.13 \pm 0.25$ & $0.213 \pm 0.019$ \\
$041006 \ldots \ldots$. & $1.915 \pm 0.016$ & $1.11 \pm 0.004$ & $0.219 \pm 0.013$ & $2.37 \pm 0.02$ & $0.092 \pm 0.001$ \\
$041016 \ldots \ldots$. & $3.263 \pm 0.288$ & $0.689 \pm 0.096$ & $1.64 \pm 0.243$ & $4.95 \pm 1.35$ & $0.7 \pm 0.031$ \\
$041127 \ldots \ldots$. & $1.809 \pm 0.091$ & $1.417 \pm 0.067$ & $0.458 \pm 0.08$ & $1.53 \pm 0.49$ & $0.341 \pm 0.038$ \\
$041211 \ldots \ldots$. & $2.804 \pm 0.188$ & $1.42 \pm 0.054$ & $1.496 \pm 0.263$ & $8.66 \pm 1.5$ & $0.389 \pm 0.029$ \\
$050123 \ldots \ldots$. & $2.013 \pm 0.092$ & $0.765 \pm 0.085$ & $0.434 \pm 0.142$ & $2.73 \pm 0.86$ & $0.395 \pm 0.045$ \\
$050209 \ldots \ldots$. & $3.311 \pm 0.274$ & $0.669 \pm 0.111$ & $1.2 \pm 0.212$ & $4.24 \pm 1.36$ & $0.663 \pm 0.043$ \\
$050408 \ldots \ldots$. & $1.778 \pm 0.083$ & $1.103 \pm 0.005$ & $0.537 \pm 0.007$ & $2.68 \pm 0.01$ & $0.261 \pm 0.002$ \\
$050509 \ldots \ldots$. & $1.104 \pm 0.345$ & $1.12 \pm 0.095$ & $-0.836 \pm 0.125$ & $0.64 \pm 0.28$ & $0.807 \pm 0.026$ \\
$050729 \ldots \ldots$. & $2.455 \pm 0.084$ & $0.172 \pm 0.059$ & $1.515 \pm 0.214$ & $4.87 \pm 0.86$ & $0.447 \pm 0.026$ \\
$050807 \ldots \ldots$. & $2.25 \pm 0.224$ & $0.781 \pm 0.117$ & $0.15 \pm 0.117$ & $1.14 \pm 0.67$ & $0.411 \pm 0.066$ \\
$050922 \ldots \ldots$. & $2.656 \pm 0.073$ & $0.285 \pm 0.062$ & $0.742 \pm 0.132$ & $4.59 \pm 0.08$ & $0.378 \pm 0.007$ \\
$051021 \ldots \ldots$. & $2.386 \pm 0.067$ & $1.184 \pm 0.053$ & $0.685 \pm 0.061$ & $3.04 \pm 0.65$ & $0.289 \pm 0.028$ \\
$051022 \ldots \ldots$. & $2.585 \pm 0.022$ & $1.995 \pm 0.012$ & $1.356 \pm 0.014$ & $3.71 \pm 0.05$ & $0.065 \pm 0.001$ \\
$051028 \ldots \ldots$. & $3.103 \pm 0.152$ & $0.509 \pm 0.1$ & $1.273 \pm 0.125$ & $6.72 \pm 2.05$ & $0.522 \pm 0.049$ \\
$051211 \ldots \ldots$. & $2.862 \pm 0.101$ & $-0.076 \pm 0.085$ & $2.24 \pm 0.377$ & $7.71 \pm 1.85$ & $0.652 \pm 0.035$ \\
$060115 \ldots \ldots$. & $2.397 \pm 0.056$ & $0.91 \pm 0.025$ & $1.096 \pm 0.065$ & $4.63 \pm 0.46$ & $0.203 \pm 0.014$ \\
$060121 \ldots \ldots$. & $2.526 \pm 0.043$ & $-0.045 \pm 0.033$ & $1.499 \pm 0.069$ & $5.37 \pm 0.62$ & $0.253 \pm 0.018$ \\
\hline
\end{tabular}

